\title{
Plasticity of brain networks in a randomized intervention trial of exercise training in older adults
}

\section{Michelle W. Voss ${ }^{1}$, Ruchika S. Prakash ${ }^{2}$, Kirk I. Erickson ${ }^{3}$, Chandramallika Basak', Laura Chaddock ${ }^{1}$, Jennifer S. Kim ', Heloisa Alves', Susie Heo ${ }^{1}$, Amanda N. Szabo ${ }^{4}$, Siobhan M. White ${ }^{4}$, Thomas R. Wójcicki ${ }^{4}$, Emily L. Mailey ${ }^{4}$, Neha Gothe ${ }^{4}$, Erin A. Olson ${ }^{4}$, Edward McAuley and Arthur F. Kramer ${ }^{4}$}

\author{
1 Department of Psychology, Beckman Institute, University of Illinois at Urbana-Champaign, IL, USA \\ 2 Department of Psychology, The Ohio State University, OH, USA \\ ${ }^{3}$ Department of Psychology, University of Pittsburgh, PA, USA \\ ${ }^{4}$ Department of Kinesiology and Community Health, University of Illinois at Urbana-Champaign, IL, USA
}

Edited by:

Lars Nyberg, Umeå University,

Sweden

Reviewed by:

Martin Lövdén, Karolinska Institutet Sweden

Jonas Persson, Stockholms universitet, Sweden

\section{*Correspondence:}

Michelle W. Voss, Department of

Psychology, Beckman Institute for

Advanced Science and Technology,

University of Illinois at Urbana-

Champaign, 405 N. Mathews Ave.,

Urbana, IL 61801, USA.

e-mail:mvoss@illinois.edu
Research has shown the human brain is organized into separable functional networks during rest and varied states of cognition, and that aging is associated with specific network dysfunctions. The present study used functional magnetic resonance imaging (fMRI) to examine low-frequency $(0.008<f<0.08 \mathrm{~Hz})$ coherence of cognitively relevant and sensory brain networks in older adults who participated in a 1-year intervention trial, comparing the effects of aerobic and nonaerobic fitness training on brain function and cognition. Results showed that aerobic training improved the aging brain's resting functional efficiency in higher-level cognitive networks. One year of walking increased functional connectivity between aspects of the frontal, posterior, and temporal cortices within the Default Mode Network and a Frontal Executive Network, two brain networks central to brain dysfunction in aging. Length of training was also an important factor. Effects in favor of the walking group were observed only after 12 months of training, compared to non-significant trends after 6 months. A non-aerobic stretching and toning group also showed increased functional connectivity in the DMN after 6 months and in a Frontal Parietal Network after 12 months, possibly reflecting experience-dependent plasticity. Finally, we found that changes in functional connectivity were behaviorally relevant. Increased functional connectivity was associated with greater improvement in executive function. Therefore the study provides the first evidence for exercise-induced functional plasticity in large-scale brain systems in the aging brain, using functional connectivity techniques, and offers new insight into the role of aerobic fitness in attenuating age-related brain dysfunction.

Keywords: exercise, aging, functional connectivity, fMRI, default mode network, executive function, aerobic fitness

\section{INTRODUCTION}

By the year 2030 one-fifth of Americans will be 65 years or older, causing health care spending to increase by an estimated $25 \%$ (He et al., 2005; CDC, 2007). Declining mental health is a primary component of increased health care costs, and a risk factor for diagnosis of Alzheimer's Disease (AD) and death (NIH, 2007; van Gelder et al., 2007). Therefore, research supporting a greater understanding of the biological basis of cognitive aging and dementia, and important factors for their prevention, is essential for the development of low-cost prevention and treatment programs to address the aging crisis. For instance, although age is a prominent risk factor for $\mathrm{AD}$, epidemiological studies have shown that lifestyle factors such as physical exercise significantly decrease age-related risks for cognitive impairment and AD (Lindsay et al., 2002; Barnes et al., 2003; Richards et al., 2003; Larson et al., 2006; Yaffe et al., 2009). The aim of this study was to further characterize potential biological mechanisms underlying restorative effects of exercise on the aging brain by examining the effect of a 1-year randomized controlled trial of exercise on age-related dysfunction in cognitively relevant brain systems and performance.
Advancing age has the greatest impact on structural integrity of the frontal and medial temporal cortices and progressively lesser impact on posterior brain regions such as the parietal and occipital cortices (O'Sullivan et al., 2001; Raz et al., 2005; Davis et al., 2009; Fjell et al., 2009). Functionally, this corresponds to disruption in brain networks that depend on efficient communication in the prefrontal cortex (Colcombe et al., 2005; Persson et al., 2006), and pathways between the frontal and posterior cortices (AndrewsHanna et al., 2007; Thomas et al., 2008). Studies also show aging is associated with altered fronto-temporal activation patterns compared to young adults during hippocampal-dependent tasks, such that older adults tend to have more fronto-temporal activation compared to younger adults who co-activate the hippocampus with regions involved in the perceptual representation of to-be remembered stimuli (Grady et al., 2003; Dennis et al., 2008). One study suggested this altered fronto-hippocampal pattern in aging might be due to deficient deactivation in a network of task-independent brain regions called the Default Mode Network (Miller et al., 2008). Thus the frontal cortex plays a prominent role in cognitive aging, and interventions that target improved communication and 
dynamic interaction between frontal and posterior and temporal brain regions are likely candidates for reducing age-related cognitive decline.

A promising approach for characterizing the nature of interactions among brain regions is to consider them as part of a larger context, or brain systems. Using functional magnetic resonance imaging (fMRI) one technique to do this is functional connectivity analysis, where functional connectivity refers to a measure of the temporal coherence between spatially remote brain regions. For instance, two regions with a positive correlation in signal over time are said to have high functional connectivity, and regions uncorrelated or negatively correlated are thought to be in separate, and sometimes competing, brain networks (Fox et al., 2005; Kelly et al., 2008). A large proportion of studies using functional connectivity, including the current study, have studied brain systems while the brain is at rest or independent of specific cognitive demands (Fox et al., 2005; Fair et al., 2007). This permits a view of the brain's intrinsic functional architecture, which is shaped continually by our experiences (Albert et al., 2009; Lewis et al., 2009) and in turn shapes how we perceive and interact with the world (Seeley et al., 2007).

Of particular interest here are brain systems affected by aging. At least three large-scale brain networks require efficient communication between the frontal cortex and the rest of the brain and thus are negatively affected by aging. These include the Default Mode Network (DMN), a fronto-executive network (FE), and a frontoparietal (FP) network. The DMN includes the posterior cingulate, ventral and superior frontal medial cortices, and bilateral lateral occipital, middle frontal, hippocampal and parahippocampal, and middle temporal cortices (Fox et al., 2005; Buckner et al., 2008). Hypotheses for the functional role of the DMN include memory consolidation, self-referential thought, mind-wandering, and autobiographical memory (Buckner et al., 2008; Schilbach et al., 2008), however evidence also supports a relationship between DMN function and executive control. For instance, increased DMN function has been associated with better working memory performance in young adults (Hampson et al., 2006), and better performance on a range of executive function tasks in older adults (Andrews-Hanna et al., 2007; Persson et al., 2007; Damoiseaux et al., 2008; Voss et al., 2010). Thus the DMN represents an important network for understanding determinants of healthy cognitive aging.

Compared to the DMN, there have been relatively few examinations relating age-associated disruption in the FE and FP networks to behavioral deficits. The FE network includes the anterior prefrontal cortex, insular and frontal operculum cortices, the tempoparietal junction, and the dorsal posterior and anterior cingulate gyri (Dosenbach et al., 2006). Functionally, this network is involved in sustained task-set maintenance, error feedback for tuning top-down control, and maintaining associations between actions and their outcomes (Rushworth et al., 2004; Dosenbach et al., 2006). Although there have been few examinations of age-related disruption in intrinsic functional connectivity in the FE network, a vast literature supports age-related decline in performing tasks implemented by this network (for review, see Park and Reuter-Lorenz, 2009).

The FP network includes the inferior parietal cortices, the supplementary motor and primary cortices, the frontal eye-fields, primary and extrastriate visual cortices, the inferior frontal cortex, and some overlapping portions of the tempo-parietal junction with the
FE network (Corbetta and Shulman, 2002; Dosenbach et al., 2006). Whereas some studies have shown age-related structural and functional disruption in the FP network (Andrews-Hanna et al., 2007; Madden et al., 2007), the relationship between network disruption in healthy aging and cognitive performance has not been clearly shown. Nevertheless, the DMN, and the FE and FP networks represent three brain systems that are susceptible to age-related disruption, likely corresponding to performance disruptions in associated functional domains of cognition. The current study focuses on the capacity for a 1-year randomized aerobic exercise program to remediate age-related disruption in these brain systems.

Our rationale for examining the effect of exercise on age-related deficits in brain connectivity stems first from evidence that aerobic exercise is associated with increased structural and functional integrity in regions that overlap with age-related brain dysfunction, including the frontal (Colcombe et al., 2003, 2004, 2006), and temporal cortices (Adlard et al., 2005; Pereira et al., 2007; Erickson et al., 2009; Creer et al., 2010). In rodents, exercise has also been shown to positively affect brain regions involved in locomotion such as the motor cortex (Kleim et al., 2002; Swain et al., 2003), the basal ganglia (MacRae et al., 1987; McCloskey et al., 2001), and the cerebellum (Black et al., 1990; Isaacs et al., 1992; Larsen et al., 2000), and this literature has primarily shown enhancements in vasculature and metabolic factors in areas directly activated by locomotion (Adkins et al., 2006). Behaviorally aerobic exercise is associated with improved performance in abilities coordinated by the frontal and temporal cortices, such as executive function and memory (Colcombe and Kramer, 2003; Colcombe et al., 2004; Pereira et al., 2007; Creer et al., 2010; Voss et al., 2010). The precise neurobiological mechanism for such cognitive effects remains unknown, however a vast rodent literature supports a central role of protective neurotrophins such as brain-derived neurotrophic factor (BDNF), nerve growth factor (NGF), and insulin-like growth factor (IGF-1) (Neeper et al., 1995, 1996; Cotman and Berchtold, 2002; Farmer et al., 2004; Vaynman et al., 2004; Gomez-Pinilla et al., 2008), which have been shown to facilitate production of new neurons in the hippocampus, promote synaptic plasticity in the hippocampus and cerebral cortex, and enhance growth and protection of neurovasculature (Chen et al., 1995; Van Praag et al., 1999a,b, 2005; Swain et al., 2003; Farmer et al., 2004; Sonntag et al., 2005; Pereira et al., 2007; Creer et al., 2010). Thus literature suggests exercise may promote formation and strengthening of connections between the hippocampus and its widespread cortical connections, including connections with the frontal and temporal cortex, and projections to the parietal and lateral occipital cortex through cingulate pathways (Miller, 1991).

Consistent with this, it has been recently shown that aerobic fitness, reflective of increased daily aerobic exercise and a physically active lifestyle, is positively associated with functional connectivity in the DMN at rest, and that this in part mediates better performance on tasks requiring set-shifting, task switching, and spatial working memory (Voss et al., 2010). That exercise is associated with improved resting or task-independent cortical function suggests exercise affects the most basic aspects of the brain's functional architecture. The DMN has also shown a growing capacity for predicting individual differences in markers of pathological aging associated with amyloid deposition (Hedden 
et al., 2009) and positive diagnosis of MCI and AD (Lustig et al., 2003; Greicius et al., 2004). Thus it appears there are interesting but relatively unexplored - links between physical activity, DMN function, cognitive aging, and risk for dementia. The current study adds to existing literature by examining exercise training-related changes in functional connectivity in the DMN, and two other brain networks known to implement complex cognition (FE and FP). To examine whether there is specificity of training effects to higher-level cognitive systems, we also examined connectivity in the motor and auditory systems. Finally, given the previous literature on aging and exercise, we assessed whether changes in functional brain organization corresponded to improved cognitive performance in tasks of verbal short-term memory (STM) and executive functions, including task-switching, set-shifting and inhibition, and spatial working memory.

Because we sought to characterize the nature of exercise training effects on the brain and cognition in aging, we first identified peak regions of age-related disruption in brain networks, based on a comparison of functional connectivity between young and elderly adults. We next identified regions that showed increased connectivity for older adults. This allowed us to determine whether exercise-related brain plasticity increases connections that are presently "in use" in older adults (i.e., do olders become increasingly different than young?), or whether exercise increases connectivity such that disruptions are remediated and brain systems shift to their original, or younger, state (i.e., do olders become more like young?). Second, we examined changes in functional connectivity among identified regions across three time-points, at baseline before training began, six months post baseline and 12 months post baseline at the conclusion of the training program. Previous research has examined the effects of six months of aerobic exercise on brain and cognition; however, to our knowledge no study has conducted a randomized controlled trial testing the effects of aerobic exercise training on human brain health over a 1-year trial. Based on the behavioral and neuroimaging effects of exercise in humans, and results from animal studies, we hypothesized that aerobic exercise training would be associated with increased functional connectivity in the frontal and temporal cortices in the DMN and FE systems, and in the frontal and parietal cortex in the FP system. We also assessed connectivity between brain regions that are involved in locomotor aspects of physical activity including the primary motor cortices. Animal studies have shown that aerobic exercise primarily enhances angiogenic factors in the motor cortex (Adkins et al., 2006); therefore if localized angiogenesis is a key factor in aerobic training-enhanced functional connectivity then we should also see functional connectivity changes in favor of the walking group in the primary motor cortex. Finally, we examined age-related decrements in the auditory system. If exercise training has a global effect on brain function and upregulation of neurotrophins, including both higher-level cognitive systems and basic sensory systems, fitness training would also enhance functional connectivity in the auditory system. However, if the effects of exercise training are specific to brain systems mediating cognitive (and perhaps motor control), then there would be no effects of exercise training on functional connectivity in the auditory system. Ultimately we believe exercise positively affects neurobiological substrates that facilitate coordination of brain systems during complex cognition (i.e., fronto and temporal connections) and in turn decreases the need for compensatory responses associated with age-related biological degradation (Park and Reuter-Lorenz, 2009). Therefore, we hypothesize that aerobic training will result in a shift toward the younger state and functional organization more like young adults.

\section{MATERIALS AND METHODS PARTICIPANTS}

Participants were recruited from the local community of UrbanaChampaign, Illinois. Eligible participants had to (1) demonstrate strong right handedness, with a $75 \%$ or above on the Edinburgh Handedness Questionnaire (Oldfield, 1971), (2) be between the ages of 18 and 35 for young adults and between 55 and 80 years for elderly adults (3) score $\geq 51$ on the modified Mini-Mental Status Exam (mMMSE, Stern et al., 1987), a screening questionnaire to rule out potential neurological pathology, (4) score $<3$ on the Geriatric Depression Scale (GDS) (Sheikh and Yesavage, 1986), (5) have normal color vision (6) have a corrected visual acuity of at least 20/40 and (7) sign an informed consent. In addition, elderly participants had to report being low in physical activity, which was defined as having been physically active for $30 \mathrm{~min}$ or more no more than two times in the last 6 months. Participants completed a mock MRI session, wherein they were screened for their ability to complete an experiment in an MRI environment. Participants who passed the mock screening subsequently completed a series of structural and functional MRI scans, and for the elderly subjects, a graded maximal exercise test. Prior to MR scanning all participants were tested for visual acuity and (if need be) corrective lenses were provided within the viewing goggles to ensure a corrected vision of at least 20/40 while in the scanner. Participants were compensated for their participation.

Demographic information for young and elderly adults is presented in Tables 1 and 2 . Elderly participants were further randomized to either an aerobic walking group or a control group that participated in a stretching and toning program. The groups did not significantly differ in baseline fitness level, mean years of education, or gender (all $p>0.05$ ), see Tables 1 and 2. Neuroimaging measures were collected as part of a larger task battery, and were originally developed to be passive viewing tasks for localizing stimulus-specific processing regions of the ventral visual cortex.

Table 1 | Participant demographics.

\begin{tabular}{|c|c|c|c|c|c|}
\hline \multirow[b]{2}{*}{ Variable } & \multirow[b]{2}{*}{$\begin{array}{l}\text { Young adult } \\
\text { control }\end{array}$} & \multicolumn{2}{|c|}{ Randomly assigned } & \multicolumn{2}{|c|}{$\begin{array}{c}p \text {-Value } \\
\text { (two-tailed) }\end{array}$} \\
\hline & & FTB control & Walkers & $\begin{array}{l}\text { Young- } \\
\text { old }\end{array}$ & $\begin{array}{l}\text { FTB- } \\
\text { walk }\end{array}$ \\
\hline$N$ & 32 & 35 & 30 & & \\
\hline Age (SD) & $23.91(4.44)$ & $65.37(5.24)$ & $67.30(5.80)$ & $* * *$ & NS \\
\hline$\%$ Female & 85 & 71 & 73 & NS & NS \\
\hline Education $^{\mathrm{a}}$ & $16.80(2.12)$ & $15.87(2.73)$ & $15.93(2.84)$ & NS & NS \\
\hline $\mathrm{mMMSE}^{\mathrm{b}}$ & na & $54.83(1.87)$ & $55.23(1.43)$ & na & NS \\
\hline
\end{tabular}

aducation refers to self-reported years of education.

${ }^{b}$ There was no main effect of change in $M M M S E$ score or group $\times$ time interaction, $p>0.05$ 
Table 2 | Fitness, BMI, and adherence for exercise groups.

\begin{tabular}{llll}
\hline & \multicolumn{3}{c}{ Mean (SD) } \\
\cline { 2 - 4 } Variable & Baseline & $\mathbf{6}$ months & $\mathbf{1 2}$ months \\
\hline FTB & & & \\
VO ${ }_{2}$ max $(\mathrm{ml} / \mathrm{kg} / \mathrm{min})^{\mathrm{a}}$ & $21.40(4.13)$ & $21.28(4.55)$ & $21.30(4.39)$ \\
$\mathrm{BMI}$ & $28.35(4.10)$ & $28.45(4.39)$ & $28.49(4.50)$ \\
Adherence & & $83.38(13.46)$ & $80.28(13.50)$ \\
WALKERS & & & \\
VO ${ }_{2}$ max $(\mathrm{ml} / \mathrm{kg} / \mathrm{min})^{\mathrm{a}}$ & $21.20(4.13)$ & $21.53(4.64)$ & $22.17(5.05)$ \\
$\mathrm{BMI}^{\mathrm{a}}\left(\mathrm{kg} / \mathrm{m}^{2}\right)$ & $28.48(4.42)$ & $28.10(4.39)$ & $28.30(4.66)$ \\
Adherence & & $78.13(14.31)$ & $76.00(14.87)$ \\
& &
\end{tabular}

${ }^{a}$ Only $\mathrm{VO}_{2}$ max had a significant linear group $\times$ time interaction effect; $p<0.05$, demonstrating that the intervention was effective in improving cardiorespiratory fitness; $B M I$, body mass index $[B M I=$ (weight in $\mathrm{kg}) /\left(\right.$ height in $\left.\left.\mathrm{m}^{2}\right)\right]$; adherence refers to percentage of exercise classes attended.

Participants in this study overlap with participants from a previously published investigation of age-related differences in stimulus processing specificity that was conducted on a subset of the crosssectional data (Voss et al., 2008). We have previously demonstrated that functional connectivity techniques are applicable to the data from a single assessment session (Voss et al., 2010). Lastly, it is important to note that participants in the current study represent a subset of all participants that completed the study, here comprised of those who completed fMRI/MRI and cognitive assessments at baseline, 6 and 12 months sessions without excessive motion or artifact in the MR data. Yet, participant demographics in the current study do not significantly differ $(p>0.05)$ from a baseline sample of elderly adults that was nearly double in sample size (Voss et al., 2010), providing evidence that participants in the current study are representative of the larger sample.

\section{MEASURES}

\section{Neuropsychological measures}

The following four tasks were used to assess verbal STM, working memory, and executive control processes, with forward and backward span assessing verbal short term memory, a spatial working memory task assessing visuo-spatial working memory, and a measure of task-switching and the Wisconsin Card Sort Task assessing executive control processes. These measures were chosen for their joint sensitivity to individual differences in aging and aerobic fitness. Details for each task are given below.

Digit span task. This task yielded two measures of STM. Forward Span was administered first. For this task, a task administrator read a sequence of numbers out loud to participants (e.g. 4-5). Participants were instructed to repeat the sequence of numbers to the administrator in the same order (e.g. 4-5). Participants were given two trials (e.g. $4-5 ; 2-4)$ for each list length. List lengths began with a two number sequence and incrementally increased by one number (maximum seven number sequence) if participants could correctly repeat the sequence in correct order for at least one trial. If participants incorrectly repeated the sequences of a particular list length in both trials, the previous list length was recorded as the dependent variable. Next, the Backward Span Task was administered. This is similar to the forward span task, except that the participants are requested to repeat the sequence of numbers back to the administrator in reverse order (e.g., 5-4, if administrator said 4-5). The number of digits increased by one (minimum 2 , maximum 7 ) until the participant consecutively failed in both trials of the same digit span length, where the previous list length, was recorded as the dependent variable. The additional requirement of holding the list in one's head while reporting the list in reverse order makes the Backward Span Task more difficult. However, backward recall lacks key components of classic working memory tasks which require continual online processing and updating of information, and has been shown to be differentially affected by aging compared to working memory and to more closely align with Forward Digit Span in patterns of age-related deficit (Bopp and Verhaeghen, 2005). Therefore, for our purposes we used performance on Forward and Backward Span as two dependent variables that represent simple and complex STM abilities, respectively.

Spatial working memory. This task provided a measure of the ability to form and retain memories of spatial locations over a delay period. Online maintenance and continual updating of spatial locations during delay and response is essential to good performance, therefore this task measures working memory abilities. First, a fixation cross-hair appeared for one second and participants were instructed to keep their eyes on the cross-hair. Following the fixation, either one, two, or three black dots appeared at random locations on the screen for a duration of $500 \mathrm{~ms}$. The dots were removed from the display and the fixation cross re-appeared on the screen for a period of $3 \mathrm{~s}$. During this time, participants were instructed to try and remember the locations of the previously presented black dots. At the end of the 3-s delay, a red dot appeared on the screen in either one of the same locations as the target dots (match condition) or at a different location (non-match condition). Participants had $2 \mathrm{~s}$ to respond to the red dot by pressing one of two keys on a standard keyboard - the ' $x$ ' key for a non-match trial, and the ' $m$ ' key for a match trial. Forty trials were presented for each set size (1,2, or 3 locations), with 20 trials as match trials and 20 trials as non-match trials. Participants were instructed to respond as quickly and accurately as possible. Several practice trials were performed before the task began in order to acquaint the participants with task instructions and responses. Since our goal was to characterize general spatial working memory performance, we computed a composite variable of reaction time and error rate across all three conditions. Normalized average reaction time and normalized average error rates were summed to create one measure summarizing overall performance in the spatial working memory task. Note due to computer error there were two participants with missing data at baseline [one walker, one flexibility, toning, and balance (FTB)].

Task-switching. This task provided a measure of executive function by testing participants' abilities to flexibly switch their focus of attention between multiple task sets. In this task participants had to switch between judging whether a number $(1,2,3,4,6,7,8$, or 9$)$ was odd or even and judging whether it was low or high (i.e., smaller or larger than 5). Numbers were presented individually for $1500 \mathrm{~ms}$ against a pink or blue background at the center of the screen, with the constraint that the same number did not appear twice in succession. 
If the background was blue, participants used one hand to report as quickly as possible whether the letter was high ("X" key) or low ("Z" key). If the background was pink, participants used their other hand to report as quickly as possible whether the number was odd ("N" key) or even ("M" key). Participants completed four single task blocks ( 2 blocks of odd/even and 2 blocks of high/low) of 24 trials each. Due to the difficulty of this task, participants were provided with a practice block in which they switched from one task to the other for 120 trials. This practice block allowed participants to become acquainted with the switching block and ensured compliance with task instructions. Finally, they completed a dual task (switching) block of 120 trials during which the task for each trial was chosen randomly. This task was similar to that of Kramer et al. (1999) and Pashler (2000); the version of the task reported in this study was programmed and administered using E-prime software (Psychology Software Tools ${ }^{1}$ ). For the current study, the primary executive function measure in this task was local switch cost, given that this was preferentially associated with aerobic fitness and functional connectivity in a cross-sectional study (Voss et al., 2010). Local switch cost refers to the difference in performance for trials when the preceding trial involved the same task (non-switch trial) and those when the preceding trial was of the other task (switch trial), and represents a measure of attentional set re-configuration and inhibition, two sub-components of executive function (Miyake et al., 2000). Only reaction time measures (based on mean reaction time) were used for computing local switch cost.

Wisconsin Card Sorting Task. In this task, participants completed a computerized version of the Wisconsin Card Sorting Task (WCST), assessing multiple components of executive function including working memory, inhibition, and switching processes (O'Sullivan et al., 2001). This task was chosen because of its reliance on executive functions and set switching, which should show similar patterns of association to the local switch cost measure described above. The participant's task was to sort cards displayed on a computer screen. The cards contained geometric designs and could be sorted into categories by shape, color, or number of the design. Participants were asked to match each card that appeared in the lower portion of the computer screen with one of four cards displayed at the top of the screen. The participants were told that the computer would provide feedback about the accuracy of their decision, but that the examiner could not give them any additional instructions about the task. For the present study, we computed a metric of perseverative errors that measures the inability to flexibly adapt to a changing rule set. The primary dependent variable for this measure was computed by taking the average of the standardized number of perseverative responses and perseverative errors, and computing their residuals from a regression predicting the total number of errors, to control for overall level of performance (Raz et al., 2003). To minimize testretest facilitation of recognizing changing rule sets, the WCST was only administered at baseline and 12-month sessions. Due to data collection error there was missing data for the WCST, including five scores at baseline and five different participants' scores at the 12-month session. For both baseline and 12-month sessions there were 2 walkers and 3 FTB participants with missing data.

${ }^{1}$ www.pstnet.com

\section{Aerobic fitness assessment}

Elderly participants were required to obtain consent from their personal physician before cardiorespiratory fitness testing was conducted. Aerobic fitness $\left(\mathrm{VO}_{2} \max \right)$ was assessed by graded maximal exercise testing on a motor-driven treadmill. The protocol involved the participant walking at a speed slightly faster than their normal walking pace (approximately $30-100 \mathrm{~m} / \mathrm{min}$ ) with increasing grade increments of $2 \%$ every $2 \mathrm{~min}$. A cardiologist and nurse continuously monitored measurements of oxygen uptake, heart rate and blood pressure. Oxygen uptake $\left(\mathrm{VO}_{2}\right)$ was measured from expired air samples taken at 30-s intervals until a maximal $\mathrm{VO}_{2}$ was attained or to the point of test termination due to symptom limitation and/or volitional exhaustion. $\mathrm{VO}_{2}$ max was defined as the highest recorded $\mathrm{VO}_{2}$ value when two of three criteria were satisfied: (1) a plateau in $\mathrm{VO}_{2}$ peak between two or more workloads; (2) a respiratory exchange ratio $>1.00$; and (3) a heart rate equivalent to their age predicted maximum (i.e., 220 - age). Due to scheduling difficulty, two participants in the stretching group did not have fitness assessments at the 6-month session; all participants had fitness assessments at baseline and 12-month sessions. $\mathrm{VO}_{2} \max$ values for participants at baseline, 6 and 12 months are shown in Table 2. Note that across all three of the time points, even with improvements from the intervention, both groups of participants were in the bottom 10th percentile of the population for $\mathrm{VO}_{2}$ max based on their age and gender (Whaley et al., 2006), reflecting our exclusive recruitment of currently low active older adults.

\section{Exercise intervention}

Older adults were randomly assigned to participate in either an aerobic walking program, or a control group that did non-aerobic stretching and toning exercises. The non-aerobic control group served to match groups for social contact associated with group exercise and to determine effects on brain function specific to aerobic exercise. Both the walking and control groups met three times per week.

Walking condition. For the walking program, a trained exercise leader supervised all sessions. Participants started by walking for $10 \mathrm{~min}$ and increased walking duration weekly by 5 -min increments until a duration of $40 \mathrm{~min}$ was achieved at week seven. Participants walked for $40 \mathrm{~min} / \mathrm{session}$ for the remainder of the program. All walking sessions started and ended with approximately 5 min of stretching for the purpose of warming up and cooling down. Participants wore heart rate monitors and were encouraged to walk in their target heart rate zone, which was calculated using the Karvonen method (Strath et al., 2000) based on the resting and maximum heart rates achieved during the baseline maximal graded exercise test. The target heart rate zone was $50-60 \%$ of the maximum heart rate reserve for weeks one to seven and $60-75 \%$ for the remainder of the program. Participants in the walking group completed an exercise log at each exercise session. Every 4 weeks, participants received written feedback forms that summarized the data from their logs. Participants with low attendance and/or exercise heart rate were encouraged to improve their performance in the following month.

Flexibility, toning, and balance control condition. For the FTB program a trained exercise leader led sessions. All FTB classes started and ended with warm-up and cool-down stretches. During each 
class, participants engaged in four muscle toning exercises utilizing dumbbells or resistance bands, two exercises designed to improve balance, one yoga sequence, and one exercise of their choice. To maintain interest, a new group of exercises was introduced every 3 weeks. During the first week, participants focused on becoming familiar with the new exercises, and during the second and third weeks, they were encouraged to increase the intensity by using more weight or adding more repetitions. Participants in the FTB group also completed exercise logs at each exercise session and received monthly feedback forms. They were encouraged to exercise at an appropriate intensity 13-15 on the Borg RPE scale; (Borg, 1985) and attend as many classes as possible.

\section{IMAGING METHODS}

For all participants, high resolution T1-weighted brain images were acquired using a 3D Magnetization Prepared Rapid Gradient Echo Imaging (MPRAGE) protocol with 144 contiguous axial slices, collected in ascending fashion parallel to the anterior and posterior commissures, echo time $(\mathrm{TE})=3.87 \mathrm{~ms}$, repetition time $(\mathrm{TR})=1800 \mathrm{~ms}$, field of view $($ FOV $)=256 \mathrm{~mm}$, acquisition matrix $192 \mathrm{~mm} \times 192 \mathrm{~mm}$, slice thickness $=1.3 \mathrm{~mm}$, and flip angle $=8^{\circ}$. All images were collected on a $3 \mathrm{~T}$ head-only Siemens Allegra MRI scanner.

Functional MRI (fMRI) scans were acquired during three passive viewing tasks: (1) a checkerboard task comprised of luminancematched flashing black-and-white checkerboards and flashing color checkerboards at a rate of $8 \mathrm{~Hz}$, each checkerboard condition was presented in two separate 30-s blocks that alternated with 20-s blocks of fixation baseline; (2) a word viewing task comprised of 30 -s blocks of words, pseudo-words, and letter strings, presented separately in two 30-s blocks that alternated with 20-s blocks of fixation baseline, each block consisted of 20 unique stimuli that were each presented for 1-s with a 500-ms fixation between each word presentation; and (3) a face/building viewing task comprised of three 20-s blocks of faces and buildings that alternated with 20-s blocks of luminance matched scrambled images (taken from the face and building stimulus set) as the baseline condition, each block consisted of 20 unique black-and-white images (controlled for luminance and dimension) that were each presented for 1-s. In each task participants were instructed to keep their eyes open and to pay attention to the screen.

Visual stimuli were presented with MRI-safe fiber optic goggles (Resonance Technologies, Inc.). Participants completed the passive viewing tasks as part of a larger battery of cognitive paradigms within the scanner. For the fMRI tasks, T2* weighted images were acquired using a fast echo-planar imaging (EPI) sequence with Blood Oxygenation Level Dependent (BOLD) contrast $(64 \times 64$ matrix, $4 \mathrm{~mm}$ slice thickness, $\mathrm{TR}=1500 \mathrm{~ms}, \mathrm{TE}=26 \mathrm{~ms}$, flip angle $=60$ ). A total of 150 volumes were acquired per participant for the checkerboard task, 220 volumes for the word task, and 180 volumes for the face/building task.

\section{IMAGE ANALYSIS}

\section{Structural MRI preprocessing}

Each participant's low-resolution EPI image was registered to his or her high-resolution T1 structural image, which was subsequently registered to stereotaxic space (study-specific template generated using $152 \mathrm{~T} 1 \mathrm{MNI}$ as the target volume, Montreal Neurological Institute) using FLIRT 12-parameter affine linear registration (Jenkinson et al., 2002). For the aging analysis a study-specific template was made including 64 representative brains chosen randomly from the sample (32 young and 32 old). This was done to ensure that the reference image was not biased toward older adults. Functional images from 6- and 12-month sessions were also registered to this study-specific template. To make the study-specific template, high-resolution structural images were first skull-stripped using BET (Smith, 2002), and manually inspected and corrected for any skull-stripping errors. Next, the structural images were registered to the $152 \mathrm{~T} 1 \mathrm{MNI}$ volume using FLIRT 12-parameter affine linear registration (Jenkinson et al., 2002). Finally, registered volumes were averaged to form a representative reference volume. Before group analyses, functional data were registered to stereotaxic space using transforms generated from the alignment of high-resolution T1 images.

\section{fMRI preprocessing}

Functional magnetic resonance imaging data preprocessing was carried out using FSL 4.1.4 (FMRIB's Software Library²). The following pre-statistics processing was applied: rigid body motion correction using MCFLIRT (Jenkinson et al., 2002), removal of non-brain structures using BET (Smith, 2002), spatial smoothing using a Gaussian kernel of FWHM 6.0-mm, grand-mean intensity normalization of the entire $4 \mathrm{D}$ dataset by a single multiplicative factor, and temporal filtering to restrict the bandwidth of the fMRI signal to $.008<f<.080 \mathrm{~Hz}$.

\section{Functional connectivity seeding analysis}

To examine age-related differences in functional connectivity, a seeding analysis was done based on known anatomical hubs of the DMN, FE, and FP networks (see Table 3). Individual-level seeding procedures followed those described in previous studies of low-frequency functional connectivity (e.g., Fox et al., 2005). The mean timeseries from three nuisance ROIs including a region in deep white matter in left temporal cortex, a region in the left lateral ventricle, and a whole brain mask to reflect global signal changes were used as nuisance regressors. Differences in heart rate variability on intrinsic fMRI BOLD changes have been associated with signal change in the CSF (de Munck et al., 2008), therefore the CSF nuisance regressor should provide protection against spurious results as a function of heart rate. Further, since the tasks involved passive viewing, we have no reason to believe they would induce individual variation in signal change related to differential task-related heart-rate modulation as a function of fitness level (e.g., van Buuren et al., 2009). Finally, whereas global signal has been shown to correlate with cardiac and respirationinduced changes in the fMRI BOLD signal (Wise et al., 2004; Birn et al., 2006; Chang et al., 2009), we have shown that there is greater sensitivity to fitness-related variation in functional connectivity when statistically controlling for global signal (Voss et al., 2010). To further isolate our examination to intrinsic functional connectivity, we also extracted signal from a bilateral ROI in primary visual cortex (125 anatomical-voxel spheres centered at $\pm 18,-98,-4$, derived from the literature (Andrews-Hanna et al., 2007)). This visual cortex regressor, along with the global signal regressor, were cautionary measures to ensure our estimates of functional connectivity were not inflated

${ }^{2}$ www.fmrib.ox.ac.uk/fsl 
Table 3 | Regions with increased functional connectivity for Young compared to Elderly adults in top-down and default mode networks.

\begin{tabular}{|c|c|c|c|}
\hline Region & $\begin{array}{l}\text { ROI } \\
\text { abbreviation }\end{array}$ & $\begin{array}{l}\text { MNI coordinates } \\
(x, y, z)\end{array}$ & $\begin{array}{l}Z \\
\text { Score }\end{array}$ \\
\hline \multicolumn{4}{|l|}{ DEFAULT MODE } \\
\hline Posterior cingulate cortex & PCC & $8,-56,30$ & $\begin{array}{l}\text { Initial } \\
\text { seed }\end{array}$ \\
\hline Frontal medial cortex & FMC & $-2,54,-12$ & 6.38 \\
\hline Left middle temporal gyrus & $\mathrm{LMTG}_{\mathrm{a}}$ & $-52,-18,-18$ & 6.00 \\
\hline Left middle frontal gyrus & LMFG & $-30,20,50$ & 5.90 \\
\hline Right middle temporal gyrus & $\mathrm{RMTG}_{\mathrm{a}}$ & $58,-10,-18$ & 5.11 \\
\hline Left parahippocampal gyrus & $\mathrm{LPHG}_{\mathrm{b}}$ & $-24,-26,-20$ & 4.03 \\
\hline Left lateral parietal cortex & $\mathrm{LLOC}_{\mathrm{c}}$ & $-44,-72,34$ & 3.63 \\
\hline Right parahippocampal cortex & $\mathrm{RPHG}_{\mathrm{b}}$ & $24,-26,-20$ & 3.25 \\
\hline Right lateral parietal cortex & $\mathrm{RLOC}_{\mathrm{c}}$ & $54,-62,32$ & 2.82 \\
\hline \multicolumn{4}{|l|}{ FRONTO-EXECUTIVE } \\
\hline $\begin{array}{l}\text { Right anterior prefrontal } \\
\text { cortex }\end{array}$ & RALPFC & $32,40,28$ & $\begin{array}{l}\text { Initial } \\
\text { seed }\end{array}$ \\
\hline Right insular cortex & $\mathrm{RINS}_{\mathrm{d}}$ & $38,4,-2$ & 5.38 \\
\hline Left prefrontal cortex & $\mathrm{LPFC}_{\mathrm{e}}$ & $-36,34,28$ & 4.90 \\
\hline Right prefrontal cortex & $\mathrm{RPFC}_{\mathrm{e}}$ & $32,42,36$ & 4.69 \\
\hline Left insular cortex & LINS $_{d}$ & $-38,8,-4$ & 4.59 \\
\hline Right inferior frontal cortex & $\mathrm{RIFG}_{f}$ & $34,48,-6$ & 4.35 \\
\hline Left inferior frontal cortex & $\mathrm{LIFG}_{f}$ & $-38,48,8$ & 3.29 \\
\hline \multicolumn{4}{|l|}{ FRONTO-PARIETAL } \\
\hline Right inferior parietal sulcus & RIPS & $25,-62,53$ & $\begin{array}{l}\text { Initial } \\
\text { seed }\end{array}$ \\
\hline Right ventral visual cortex & $\mathrm{RVIS}_{g}$ & $36,-62,0$ & 6.94 \\
\hline Left ventral visual cortex & LVVIS $_{g}$ & $-44,-62,-6$ & 5.82 \\
\hline $\begin{array}{l}\text { Right frontal opercular/insular } \\
\text { cortex }\end{array}$ & RFOI & $28,26,8$ & 5.12 \\
\hline Right supramarginal gyrus & RSMG & $32,-38,38$ & 3.58 \\
\hline Right lateral occipital cortex & RLOC & $26,-64,54$ & 3.51 \\
\hline
\end{tabular}

ROIs with like subscripts were combined to make bilateral ROIs. See Figure 1 for anatomical visualization of ROIs on PALS-B12 atlas; seed-to-ROI age effects were largely within networks, thus inter-regional relationships for all ROI pairs (where bilateral ROls taken as single ROI) were examined for subsequent fitness effects.

due to the additive influence of synchronized task-evoked signal change. In addition to nuisance fMRI signal, six motion parameters computed by rigid body translation and rotation in preprocessing (Jenkinson et al., 2002) were included in the nuisance regression.

Following nuisance regression, normalized residual functional volumes were used for extracting a corrected mean timeseries of seeds of interest from individual functional runs. Voxel-wise Pearson correlation coefficients between the corrected timeseries of the seed region and the timeseries of each voxel in the residual image were computed in MATLAB. These statistical maps were converted to Fisher's Z maps using Fisher's r-to-z transformation (Zar, 1996) to improve normality. This procedure was done for each passive viewing task. Individual-level analyses of voxel-wise functional connectivity were then aggregated within subject (across the three functional runs) for greater statistical power, using ordinary least squares (OLS) in FSL's FEAT tool. Finally, the mean individual-level statistical maps were forwarded to a mixed-effects OLS group analysis that considered between-subject variation (Beckmann et al., 2003). Aggregating data from three passive viewing tasks, in addition to providing greater statistical power, ensures that the results are independent of ongoing visual stimulation. Furthermore, functional connectivity estimates in young adults showed comparable (within 1 standard deviation) test-retest reliability with pure resting state data (Shehzad et al., 2009), suggesting the data have comparable reliability to other resting state studies. The DMN was examined in Shehzad et al., so following the protocol outlined in their study we calculated intra-class correlations (ICC) across the three runs among ROI pairs showing age-disruption in the DMN (see Table 3). In young adults $\mathrm{ICC}=0.32(\mathrm{SD}=0.11)$ and in older adults $\mathrm{ICC}=0.18$ $(S D=0.10)$. Note ICC's were only calculated on young adults in Shehzad et al. (2009); to our knowledge no published ICC estimates exist for resting state data of healthy older adults.

In the mixed-effects between-subjects analysis, we compared young and elderly adults' mean functional connectivity, using five initial seeds to anchor the three cognitive networks of interest (see Table 3) and two sensory networks (see Table 5), while (in a voxelwise manner) controlling for the variance associated gray matter volume. The Young > Old contrast of interest resulted in a statistical map representing where aging is associated with decreased functional connectivity with an anchored network seed (see Tables 3 and 5). ROIs were determined from this analysis based on statistical peaks in separable anatomical brain areas known to comprise each network and as demarcated in the Harvard-Oxford cortical atlas packaged with the FSL software package (FSL 4.1.4, FMRIB's Software Library $y^{3}$ ). For the cognitive networks, a parallel analysis was done to identify regions where older adults had greater connectivity than younger adults. Peaks for all contrasts are listed in Tables 3-5 and are illustrated in Figure $\mathbf{1}$ for the cognitive networks. Note, following mixed-effects higher-level analysis, the distribution of statistical parametric maps was determined as Gaussian and statistical thresholding was based on Z-statistic scores of contrast parameter estimates; statistical map threshold was set at voxel $Z=2.33$, with cluster correction of $p<0.05$ (Worsley et al., 1992).

Cross-correlation of timecourses between peak ROIs was computed for each participant, fisher transformed to ensure additivity, and then averaged across the three runs. Fisher transformed correlation estimates from within network ROI-pairs were the primary dependent measures used to examine the treatment effect on age-related dysfunction in functional connectivity. Unlike the ROIs derived from the Young > Old contrast, the Old > Young contrast resulted in ROIs from networks mostly distinct from the seed (see Table 4 and Figure 1). Therefore, all inter-regional relationships were not representative of connectivity that was greater for older compared to young adults. Consequently, we only examined direct seed-to-ROI pairs for the analysis of exercise effects on connectivity in regions where old had greater baseline connectivity compared to young.

\section{Statistical analyses}

Intervention effect on functional connectivity. A series of multivariate analysis of covariance analyses (MANCOVA) were conducted to determine the effects of exercise training (walking, FTB) on

${ }^{3}$ www.fmrib.ox.ac.uk/fsl 


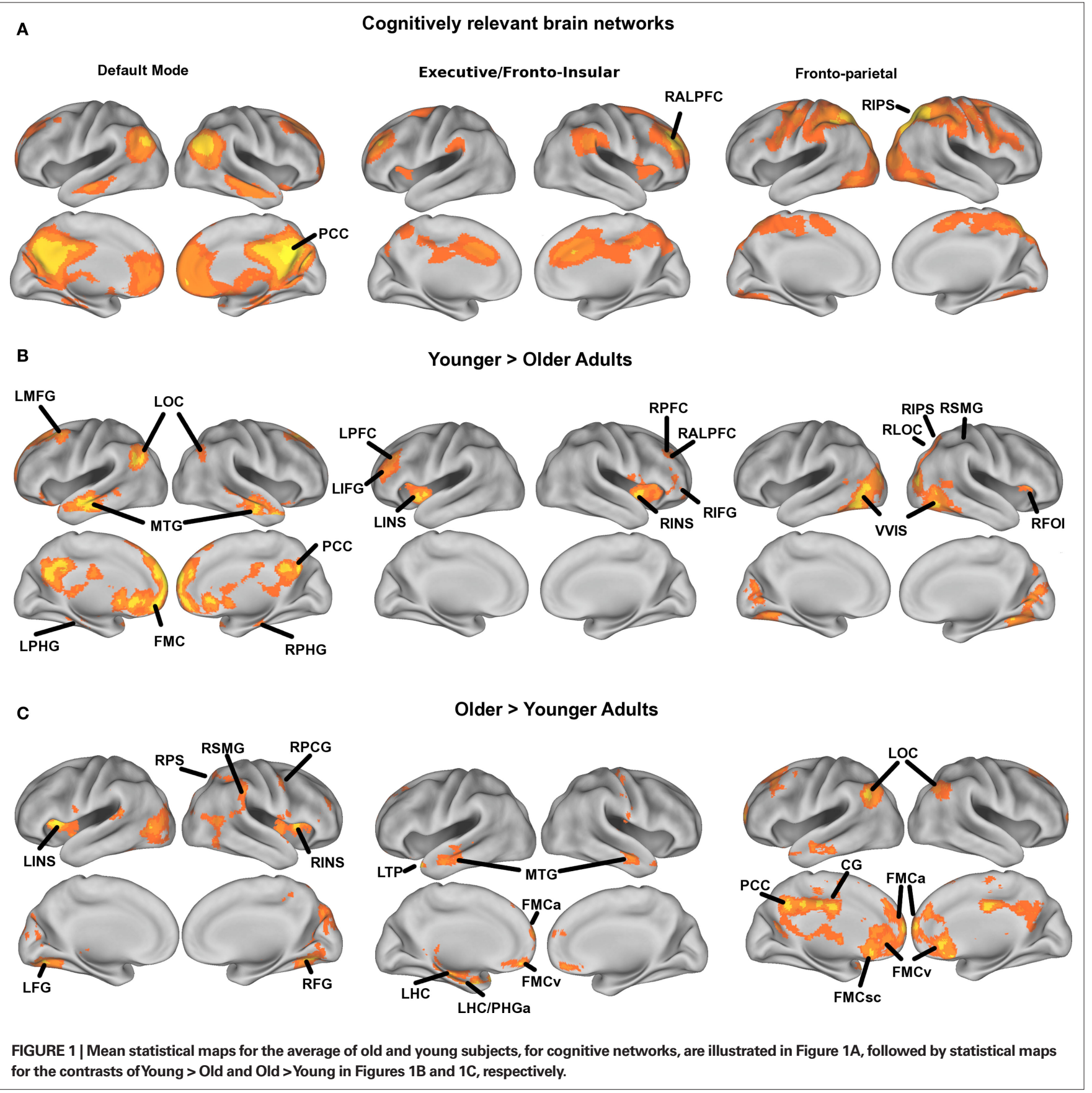

dependent measures of functional connectivity. Separate analyses were conducted for each network to assess (a) change from baseline to the 6-month session, (b) change from baseline to the 12-month session, and (c) when there was a group difference for either the 6 or 12-month session, we assessed change from the 6 to 12 -month session. The last step was done to characterize the temporal nature of the magnitude of change between sessions. Furthermore, the ANCOVA analysis approach was chosen over repeated measures analysis due to observation of chance group differences at baseline in some ROI-pairs (not all of which ended up showing statistically significant change effects), and ANCOVA provides a comparison of groups that is unaffected by baseline differences (Vickers and Altman, 2001). That is, since one disadvantage of repeated measures is that a group $\times$ time interaction can be driven by several factors, some of which may not be relevant to the question of interest (such as group differences at baseline), this analysis method may have led to spurious interaction effects in the current study (Vickers and Altman, 2001; Vickers, 2005).

First, functional connectivity of ROI pairs that showed agerelated disruption in the DMN at baseline were extracted at the 6-month session and entered as multivariate dependent variables, and examined as a function of intervention group, while baseline 
Table 4 | Regions with increased functional connectivity for Olders compared to Young adults in top-down and default mode networks.

\begin{tabular}{|c|c|c|c|}
\hline Region & $\begin{array}{l}\text { ROI } \\
\text { abbreviation }\end{array}$ & $\begin{array}{l}\text { MNI coordinates } \\
(x, y, z)\end{array}$ & $\begin{array}{l}Z \\
\text { Score }\end{array}$ \\
\hline \multicolumn{4}{|l|}{ DEFAULT MODE } \\
\hline Posterior Cingulate Cortex & PCC & $8,-56,30$ & $\begin{array}{l}\text { Initial } \\
\text { seed }\end{array}$ \\
\hline Left insular cortex & LINS $_{a}$ & $-32,12,4$ & 5.43 \\
\hline Right insular cortex & RINS $_{a}$ & $30,14,4$ & 5.01 \\
\hline Right parietooccipital sulcus & RPS & $14,-82,48$ & 4.69 \\
\hline Left occipital fusiform gyrus & $\mathrm{LFG}_{\mathrm{b}}$ & $-16,-80,-8$ & 4.53 \\
\hline Right occipital fusiform gyrus & $\mathrm{RFG}_{\mathrm{b}}$ & $26,-62,-8$ & 4.41 \\
\hline Right supramarginal gyrus & RSMG & $62,-34,44$ & 4.23 \\
\hline Right precentral gyrus & RPCG & $40,-4,38$ & 3.99 \\
\hline \multicolumn{4}{|l|}{ FRONTO-EXECUTIVE } \\
\hline $\begin{array}{l}\text { Right anterior prefrontal } \\
\text { cortex }\end{array}$ & RALPFC & $32,40,28$ & $\begin{array}{l}\text { Initial } \\
\text { seed }\end{array}$ \\
\hline Left temporal pole & LTP & $-44,20,-24$ & 5.54 \\
\hline Right middle temporal gyrus & $\mathrm{RMTG}_{\mathrm{a}}$ & $70,-14,-16$ & 5.27 \\
\hline $\begin{array}{l}\text { Left hippocampus/anterior } \\
\text { parahippocampal gyrus }\end{array}$ & $\mathrm{LHC} / \mathrm{PHGa}$ & $-16,-8,24$ & 5.24 \\
\hline Left hippocampus & $\mathrm{LHC}$ & $-24,-22,-18$ & 4.94 \\
\hline Ventral frontal medial cortex & FMCV & $-8,54,-18$ & 4.82 \\
\hline Anterior frontal medial cortex & $\mathrm{FMCa}$ & $-12,66,18$ & 4.66 \\
\hline Left middle temporal gyrus & LMTG $_{a}$ & $-56,-10,-22$ & 4.03 \\
\hline \multicolumn{4}{|l|}{ FRONTO-PARIETAL } \\
\hline Right inferior parietal sulcus & RIPS & $25,-62,53$ & $\begin{array}{l}\text { Initial } \\
\text { seed }\end{array}$ \\
\hline Left lateral occipital cortex & $\mathrm{LLOC}_{\mathrm{a}}$ & $-56,-68,36$ & 5.93 \\
\hline $\begin{array}{l}\text { Right subcallosal frontal } \\
\text { medial cortex }\end{array}$ & FMCsc & $2,20,-22$ & 5.69 \\
\hline Right lateral occipital cortex & $\mathrm{RLOC}_{\mathrm{a}}$ & $58,-60,38$ & 5.32 \\
\hline Cingulate gyrus & CG & $0,-10,32$ & 5.16 \\
\hline Posterior cingulate gyrus & PCC & $-10,-54,36$ & 5.04 \\
\hline Frontal medial cortex & FMC & $2,44,18$ & 4.94 \\
\hline Ventral frontal medial cortex & FMCV & $0,48,0$ & 4,85 \\
\hline
\end{tabular}

ROIs with like subscripts were combined to make bilateral ROIs; see Figure 1 for anatomical visualization of ROIs on PALS-B12 atlas; since regions in this contrast were not part of the seed's network, only the seed-to-ROI relationships were examined for subsequent fitness effects.

functional connectivity in the same ROI pairs were entered as covariates. Following the test for 6-month effects, the same analysis was done for 12-month effects using functional connectivity from ROI pairs from the 12-month session as dependent variables and ROIs pairs from baseline as covariates. Given a significant group difference at either the 6 or 12-month session, the same analysis was done for 12-month effects using functional connectivity from ROI pairs from the 12-month session as dependent variables and ROIs pairs from the 6-month session as covariates. This procedure was repeated for the FE, FP, and primary motor and auditory networks for Young > Old contrast, and for the DMN, FE, and FP networks for the Old > Young contrast. The sensory networks were not tested for the Old > Young contrast because it is thought that compensatory neural circuits most likely develop under high cognitive load in aging, and therefore should be manifest most in neural networks that support cognitively demanding mental activity. Thus we based our investigation of the Old > Young contrast in the context of the cognitive networks. As evident in Figure 1, older adults showed greater connectivity between rather than within networks. As a result, ROI pairs other than those including the initial seed were often not representative of pairs with greater connectivity for older adults. Therefore, we examined exercise-induced change in regions where older adults had greater connectivity at baseline by only including ROI pairs that included the initial seed. Otherwise, the procedure for examining changes in functional connectivity where olders initially had more connectivity was the same as described above. For all tests a non-significant Levene's test confirmed that assumptions of homogeneity of variance were met, and are reported if found otherwise. Overall model fit was assessed in each network using Pillai's Trace, a conservative metric for two-group comparisons, followed by univariate ANOVA tests for each ROI pair. For all analyses, unless otherwise noted, effects were considered statistically significant if $p<0.05$. Exercise effects are visualized by plotting marginal group means from the analysis at the corresponding time point (error bars represent $1 \pm$ standard error of the marginal mean). Statistical analyses were conducted using SPSS 17.0 for Macintosh.

Effects of intervention on cognition. To examine whether cognitive performance changed as a function of intervention we computed composite measures of cognitive performance based on an exploratory principle components analysis (PCA). PCA was conducted on the five primary cognitive measures (forward span, backward span, spatial working memory, local switch cost, perseverative errors) with orthogonal rotation (varimax). Components were determined by grouping individual measures with the component for which they had the largest loading, given an overall component eigenvalue greater than one. The correlation matrix between measures was also examined to ensure the correlation between measures was consistent with the determined components. Following PCA at baseline, we generated composite cognitive measures by normalizing the scores associated with each measure and summing measures that loaded on common components. Since the WCST was only administered at baseline and 12-month sessions, cognitive composite scores were only computed for the baseline and 12-month session. Finally, we assessed whether the intervention had an effect on cognition by doing a repeated measures analysis with cognitive composite measures from the 12-month session as the within-subject factors (repeated measures), group as the between-subjects factor, and baseline cognitive composite measures as covariates.

Linking biological and cognitive change. To determine whether changes in functional connectivity corresponded to meaningful change in cognitive performance, we conducted partial correlations between cognitive composite measures from the 12-month session and functional connectivity measures from the 12-month session (including only ROI-pairs that showed intervention effects in favor of either group), while controlling for variance in their respective baseline measures. 


\section{RESULTS \\ AGE DIFFERENCES IN FUNCTIONAL CONNECTIVITY}

Mean statistical maps for the average of old and young subjects, for cognitive networks, are illustrated in Figure 1A, followed by statistical maps for the contrasts of Young $>$ Old and Old $>$ Young in Figures 1B and 1C, respectively. Functional anatomical coordinates and description of ROI abbreviations corresponding to peak age differences are listed in Table 3 (Young > Old) and Table 4 (Old $>$ Young). We also tested for age-disruption in two sensory networks, corresponding to primary motor and auditory systems. Statistical peaks and description of ROI abbreviations for the Young > Old contrast in these networks is listed in Table 5.

\section{TRAINING EFFECTS ON CHANGES IN FUNCTIONAL CONNECTIVITY}

Multivariate analysis of covariance was conducted to determine the effects of training (walking, FTB) on dependent measures of functional connectivity.

\section{Default mode network}

After 6 months of training we found a non-significant, but trending, effect on the connectivity of the DMN, Pillai's trace $V=0.42, F(15,34)=1.66, p=0.11, \eta^{2}=0.42$. However, regionally the effects were in favor of the FTB group. Univariate ANOVAs revealed significant ROI pairs for connectivity between PCC-FMC, $F(1,48)=4.17, p<0.05, \eta^{2}=0.08$, and PCC-BMTG, $F(1,48)=4.11$, $p<0.05, \eta^{2}=0.08$ (see Figures 2A,B). Neither the PCC-FMC nor PCC-BMTG ROI pair showed a significant change from the 6 to 12 -month session, $p>0.05$.

After 12 months of training there was a trend in favor of the walking group for DMN connectivity, Pillai's trace $V=0.43$, $F(15,34)=1.72, p=0.09, \eta^{2}=0.43$. Univariate ANOVAs revealed

Table 5 | Regions with increased functional connectivity for Young compared to Elderly adults in sensorimotor and auditory cortex.

\begin{tabular}{|c|c|c|c|}
\hline Region & $\begin{array}{l}\text { ROI } \\
\text { abbreviation }\end{array}$ & $\begin{array}{l}\text { MNI coordinates } \\
(x, y, z)\end{array}$ & $\begin{array}{l}Z \\
\text { Score }\end{array}$ \\
\hline \multicolumn{4}{|l|}{ MOTOR SYSTEM } \\
\hline Right precentral gyrus & RMOT & $32,-10,52$ & $\begin{array}{l}\text { Initial } \\
\text { seed }\end{array}$ \\
\hline Left occipital fusiform gyrus & LFG & $-30,-68,0$ & 5.71 \\
\hline Left inferior temporal gyrus & LITG & $-42,-46,-16$ & 4.60 \\
\hline Right insular cortex & $\mathrm{RIC}$ & $34,-4,10$ & 4.57 \\
\hline $\begin{array}{l}\text { Right temporal fusiform } \\
\text { cortex }\end{array}$ & RTF & $40,-26,-20$ & 4.43 \\
\hline Left putamen & LPUT & $-28,-4,14$ & 4.41 \\
\hline \multicolumn{4}{|l|}{ AUDITORY SYSTEM } \\
\hline Right heschl's gyrus & RAUD & $46,-18,8$ & $\begin{array}{l}\text { Initial } \\
\text { seed }\end{array}$ \\
\hline $\begin{array}{l}\text { Left central opercular cortex/ } \\
\text { heschl's gyrus }\end{array}$ & LCO & $-50,-20,14$ & 5.63 \\
\hline Left cingulate gyrus & LCG & $-4,-16,44$ & 4.80 \\
\hline Right heschl's gyrus & $\mathrm{RHG}$ & $54,-20,12$ & 4.50 \\
\hline Left insular cortex & LIC & $-34,-4,12$ & 4.37 \\
\hline Left superior frontal gyrus & LSFG & $-24,-8,64$ & 4.29 \\
\hline
\end{tabular}

Age-related disruption in these ROIs was not sensitive to fitness intervention.

\section{Functional connectivity changes in favor of the FTB group}

A

B
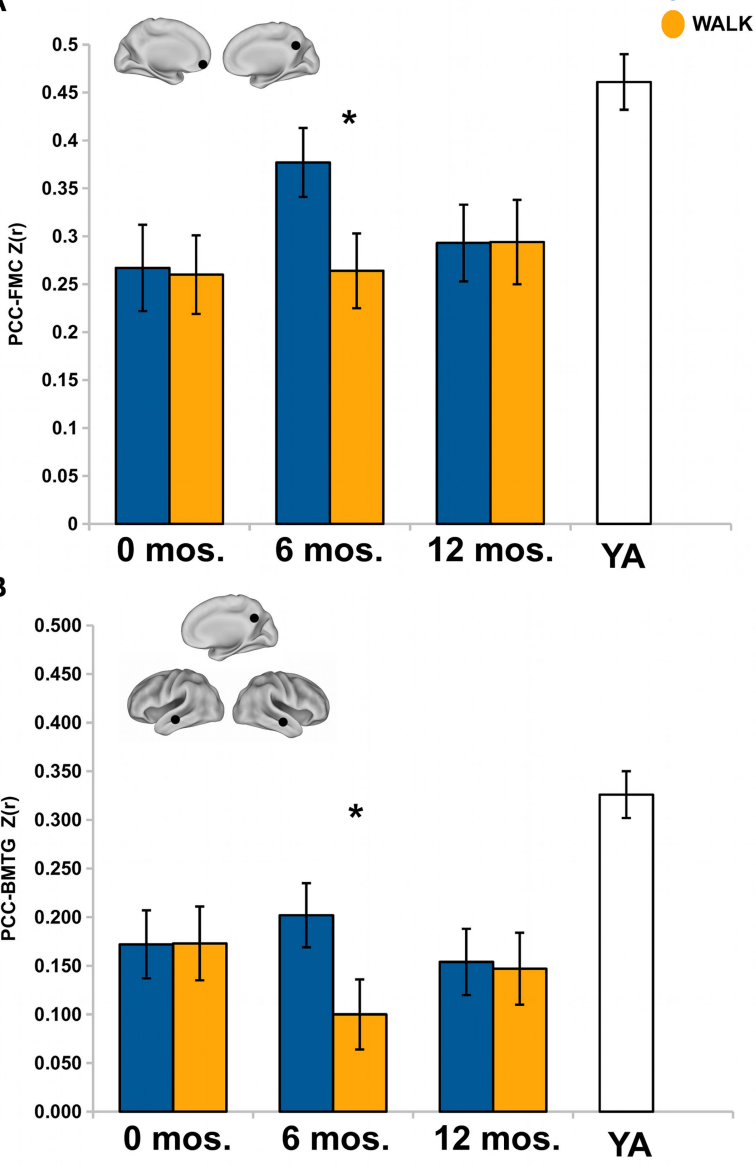

C

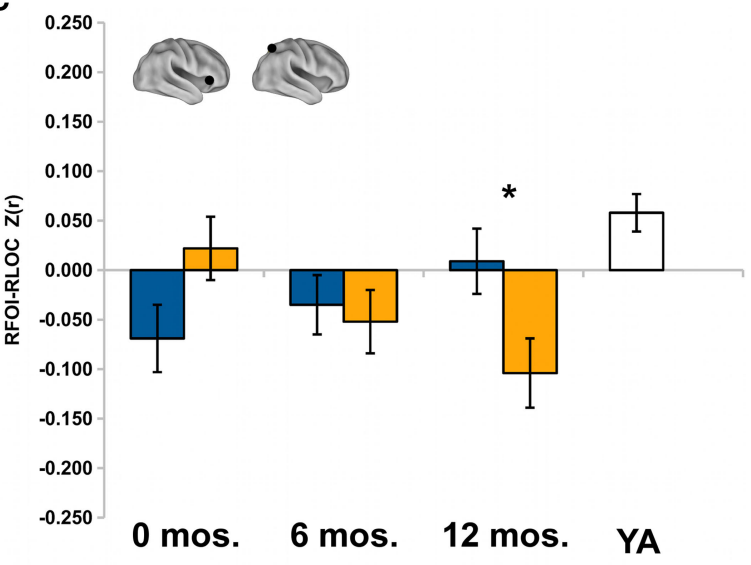

FIGURE 2 | Significant effects in favor of the FTB group, in regions reflecting age-related network disruption, in the DMN. (A, B) and the FP network (C), are visualized by plotting marginal group means from the analysis at the corresponding time point (error bars represent $1 \pm$ standard error of the marginal mean); ${ }^{*} p<0.05$. Refer to Table $\mathbf{3}$ for anatomical description and $\mathrm{MNI}$ coordinates of ROls represented by black circles. For all brains, $R=R$ and $\mathrm{L}=\mathrm{L}$. 
significant ROI pairs, all in favor of the walking group, for connections locally in the medial temporal lobe [BMTG-BPHG: $\left.F(1,48)=9.81, p<0.05, \eta^{2}=0.17\right]$, between the medial temporal lobe and the lateral occipital cortex (BPHG-BLOC: $F(1,48)=4.26$, $p<0.05, \eta^{2}=0.08$ ), and between the middle temporal gyrus and the prefrontal cortex [LMFG-BMTG: $\left.F(1,48)=4.31, p<0.05, \eta^{2}=0.08\right)$ (see Figures 3A-C). One ROI pair, BPHG-BLOC also showed significant change from 6 to the 12 -month session, $F(1,48)=4.75$, $p<0.05, \eta^{2}=0.09$, and another ROI pair involving the medial temporal cortex, BMTG-BPHG, showed marginal change from the 6 to 12 -month session, $F(1,48)=3.52, p=0.07, \eta^{2}=0.07$. Note although the other ROI pair, LMFG-BMTG did not significantly change from 6 to 12 months, the data revealed a similar trend for increasing change (Figure 3C).

For regions that showed greater baseline connectivity for older adults compared to younger adults, we examined training-related change in only the seed-to-ROI pairs. After 6 months of training, there was no overall effect of group on connectivity between the PCC and aging-associated ROIs, Pillai's Trace $V=0.08$, ns, $\eta^{2}=0.08$; separate univariate ANOVAs revealed no regional effects. Similarly, after 12 months of training, group was not associated with change in aging-associated ROIs, Pillai's Trace $V=0.06$, ns, $\eta^{2}=0.06$; separate univariate ANOVAs revealed no regional effects.

\section{Frontal executive network}

After 6 months of training, there was no overall effect of group on connectivity in the FE network, Pillai's Trace $V=.07$, ns, $\eta^{2}=0.07$. Separate univariate ANOVAs revealed a marginally significant effect in favor of the walking group for the INS-PFC connection, $F(1,57)=2.90, p=0.09, \eta^{2}=0.05$.

After 12 months there was no overall effect of group on functional connectivity in the FE network, Pillai's Trace, $V=0.14$, ns, $\eta^{2}=0.14$. Separate univariate ANOVAs indicated only one significant ROI pair, in favor of the walking group, RALPFC-PFC, $F(1,57)=6.19$, $p<0.05, \eta^{2}=0.10$ (see Figure 3D). Although the RALPFC-PFC ROI pair showed a pattern of increasing connectivity from 6 to 12 months, the change was not statistically significant, $p>0.05$.

For regions that showed greater baseline connectivity for older adults compared to younger adults, after 6 months of training there was no overall effect of group, Pillai's Trace $V=0.05$, ns,

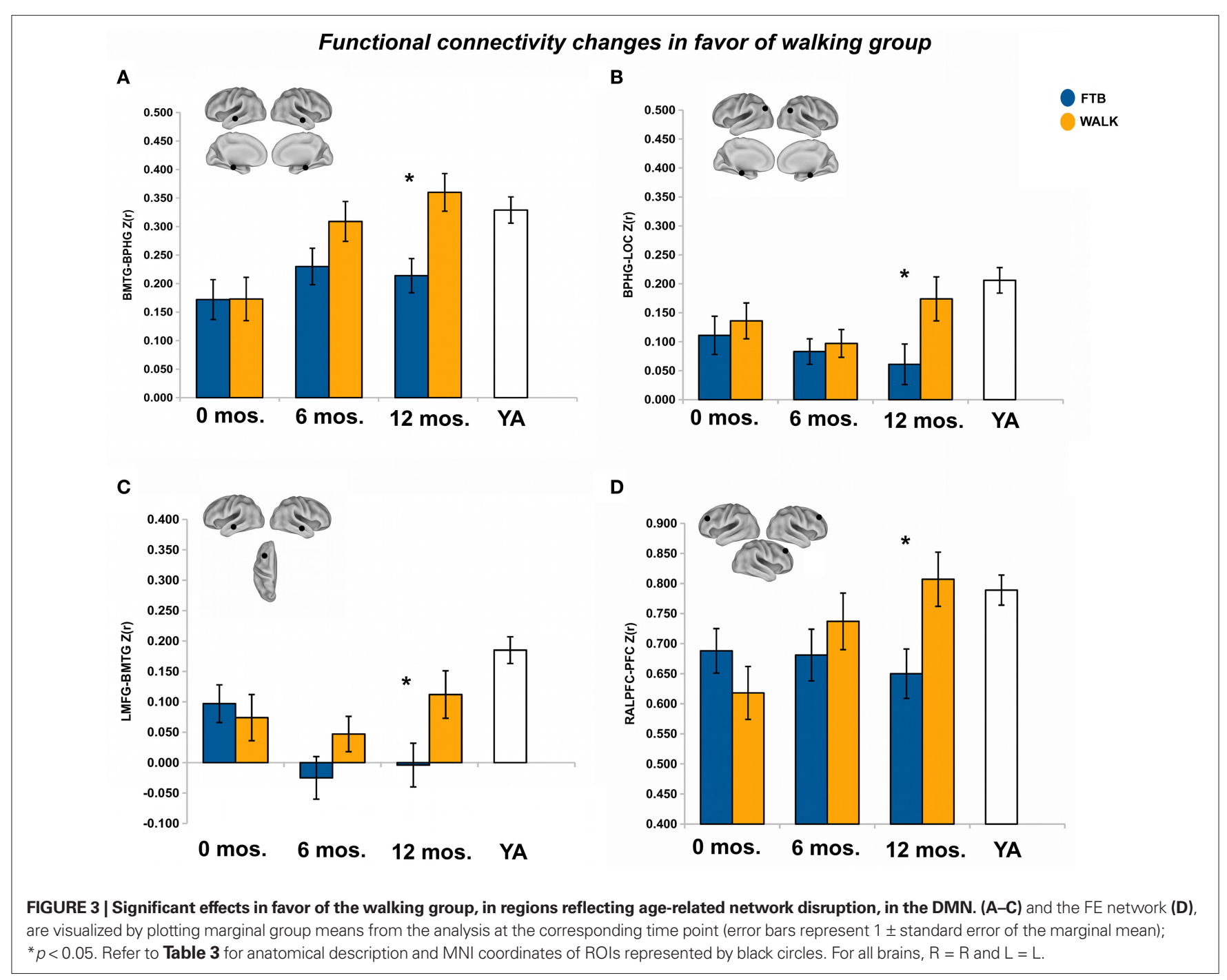


$\eta^{2}=0.05$; separate univariate ANOVAs revealed no regional effects. After 12 months of training, group was not associated with change in aging-associated ROIs, Pillai's Trace $V=0.11$, ns, $\eta^{2}=0.11$; separate univariate ANOVAs revealed a regional effect of an increasing negative correlation in the RALPFC-LHC/APHG connection for walkers, $F(1,57)=4.25, p<0.05, \eta^{2}=0.07$ (see Figure 4). In addition, there was significant change in connectivity from the 6 to 12 -month session, $F(1,57)=5.38, p<0.05$, $\eta^{2}=0.09$.

\section{Fronto-parietal network}

At 6 months, there was no overall effect of group on connectivity in the FP network, Pillai's Trace $V=0.12$, ns, $\eta^{2}=0.12$; univariate ANOVAs revealed no regional effects.

After 12 months of training, there was no overall effect of group on connectivity in the FP network, Pillai's Trace, $V=0.22$, $\mathrm{ns}, \eta^{2}=0.22$. Separate univariate ANOVAs indicated one significant ROI pair, in favor of the FTB group, RFOI-RLOC: $F(1,53)=5.32$, $\left.p<0.05, \eta^{2}=0.09\right)$ and another ROI pair with marginal significance in favor of the FTB group, RIPS-RFOI: $F(1,53)=3.60, p=0.06$, $\left.\eta^{2}=0.06\right)$. Connectivity in the RFOI-RLOC pair showed marginal change from the 6 to 12 -month session, $F(1,53)=2.78, p=0.10$, $\eta^{2}=0.05$ (see Figure 2C).

For regions that showed greater baseline connectivity for older adults compared to younger adults, after 6 months of training, there was no overall effect of group, Pillai's Trace $V=0.11$, ns, $\eta^{2}=0.12$; separate univariate ANOVAs revealed no regional effects. Similarly, after 12 months of training, there was no overall effect of group, Pillai's Trace $V=.04$, ns; $\eta^{2}=0.04$; separate univariate ANOVAs revealed no regional effects.

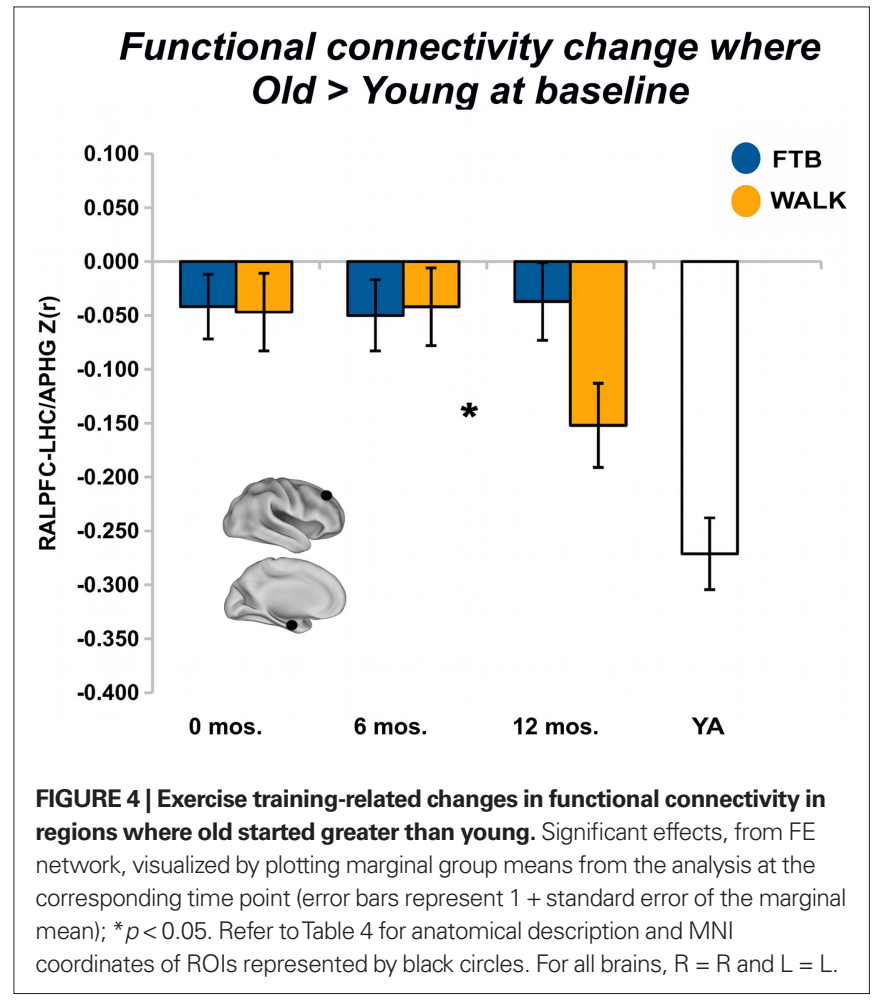

\section{Primary motor cortex}

In light of animal literature that has demonstrated exercise-dependent plasticity and angiogenesis in the motor cortex, we examined whether exercise training was associated with age-sensitive decline in connectivity with the right primary motor cortex. Age-sensitive disruptions with the right motor cortex are listed in Table 5. Analysis of training effects revealed no effect of change in connectivity with the motor system as a function of exercise group. At 6 months, Pillai's Trace $V=0.04$, ns, $\eta^{2}=0.04$; separate univariate ANOVAs revealed no regional effects. At 12 months, Pillai's Trace $V=.03$, ns, $\eta^{2}=0.03$; separate univariate ANOVAs revealed no regional effects.

\section{Primary auditory cortex}

We also examined whether exercise training was associated with age-sensitive decline in connectivity with the right primary auditory cortex. Age-sensitive disruptions with the right auditory cortex are listed in Table 5. Analysis of training effects revealed no effects of change in connectivity with the auditory system as a function of exercise group. At six months, Pillai's Trace $V=.03$, ns, $\eta^{2}=.03$; separate univariate ANOVAs revealed no regional effects. At 12 months, Pillai's Trace $V=0.09$, ns, $\eta^{2}=0.09$; separate univariate ANOVAs revealed no regional effects.

\section{TRAINING EFFECTS ON CHANGES IN COGNITIVE PERFORMANCE PCA on baseline cognitive measures}

Rotated factor loadings are shown in Table 6. The Kaiser-MeyerOlkin measure verified sampling adequacy for the analysis, $\mathrm{KMO}=0.58$, and Bartlett's Test of sphericity $\chi^{2}(10)=26.75$, $p<0.05$, indicated that the correlations between measures were sufficiently large for PCA. The measures that cluster on the same components suggest that component 1 represents a measure of executive control including measures of working memory (spatial working memory), task-switching (local switch cost), and cognitive flexibility and inhibition (perseverative errors), while component 2 represents an index of short-term verbal memory (forward and backward digit span).

For the composite measure of executive control (EXEC), normalized measures of spatial working memory, local switch cost, and perseverative errors were summed and multiplied by negative 1 so that a higher composite score reflected better performance. Normalized measures of forward and backward digit span were summed to create a composite measure of STM. Repeated measures analysis of composite measures (STM, EXEC) from the 12-month session showed a non-significant trend of improved performance for the walking group compared to the FTB group, main effect of group $F(1,46)=2.42, p=0.12, \eta^{2}=0.05$.

Table 6 | Principle component loadings of primary cognitive measures at baseline assessment; only component loadings $>0.40$ are shown.

\begin{tabular}{lll}
\hline & Component 1 & Component 2 \\
\hline Forward span & & 0.872 \\
Backward span & & 0.816 \\
Spatial working memory & 0.715 & \\
Local switch cost & 0.660 & \\
Perseverative errors & 0.734 &
\end{tabular}




\section{Linking change in functional connectivity to change in performance}

Given that the walking group improved more in both cognitive factors, a positive correlation would favor the walking group. To protect against multiple tests, we used a Bonferonni corrected $p$-value of $p<0.008$ (i.e., correction over tests for six ROI pairs) for reporting significant correlations. Results showed that functional connectivity between BPHG-BLOC in the DMN was correlated with better executive function performance $\operatorname{pr}(44)=.39, p=0.003$. We also found a trend for increased connectivity between LMFG-BMTG in the DMN as positively correlated with better executive function performance $\operatorname{pr}(44)=0.27, p=0.03$.

\section{DISCUSSION}

This study demonstrated that moderate exercise enhances functional connectivity between regions with age-related disruption in cognitively relevant brain networks. We found that the walking group showed increased connectivity after 12 months in regional connections supporting the DMN and FE systems, particularly in the frontal and temporal cortices, while the FTB group showed increased connectivity in regions of the DMN and the FP system at 6 and 12 months, respectively. Interestingly, regions sensitive to age-related disruption showed patterns of change such that older adults showed increased connectivity to become more like young adults. This pattern occurred for both the FTB and walking groups, and may suggest that observed changes in functional connectivity reflected restoration of age-degraded neural circuits. And while we did see this pattern of change support improved executive function, results were limited.

However, the pattern of connections in the DMN, FE, and FP networks that were greater for older adults compared to younger adults (see Figure 1C) suggests that with age there is decreased specificity, or dedifferentiation, of large-scale networks. Dedifferentiation of neural systems has been proposed as at least one functional determinant of the need for compensatory brain activity in older adults (Park and Reuter-Lorenz, 2009). Another proposed determinant of compensatory brain activation in older adults is an inability to suppress DMN activity during higher-level cognitive tasks. For example, attenuated task-induced suppression of the DMN has been coupled to delayed over-activation in task-relevant networks (Persson et al., 2007). In this context, it is interesting that we found with increasing age the DMN shows increased connectivity with aspects of both the FE and the FP networks, while the FE network showed increased connectivity specifically with the temporal cortices and the FP network showed increased connectivity with primarily DMN components (see Figure 1C). This is consistent with previous literature that has shown increased frontal activation for tasks that are dependent on hippocampal activity (Persson et al., 2006; Dennis et al., 2008), and dysregulation of the DMN in transition periods between rest and task that depend on reorienting attention to the task set (Persson et al., 2007; Miller et al., 2008). However, we found that aerobic exercise training led to an increased negative correlation between the right anterior prefrontal cortex in the FE network and a region in the left hippocampus just anterior to the hippocampal region found in the DMN in this study. This suggests aerobic exercise training increased the task-independent differentiation between the executive and the DM networks. We speculate this would result in greater regulation of interactions between the DMN and task networks, resulting in less over-activation during effortful memory tasks. Yet to really understand whether this would translate to improved brain function under cognitive demand, and to relate this finding more directly to theories of over-activation in aging, future research should examine such changes with respect to changes in task-evoked activation and performance.

To understand the behavioral relevance of exercise-induced changes in functional connectivity, we assessed the association between changes in connectivity in ROI pairs that showed intervention effects in favor of both groups and cognitive performance. Results showed that changes in connectivity in the DMN were associated with improved executive function performance. It may seem curious that the DMN had stronger associations with executive function performance than connectivity between the left and right prefrontal cortices in the FE system. However, it is generally still unclear how individual differences in cognitive performance are associated with resting state connectivity in the task networks such as the FE and FP. Presumably network disruption in task networks reflects a fundamental disruption present when the network is in the active state. However, resting state within-network connectivity would not necessarily predict how well an individual could implement compensatory strategies or coordinate flexible between-network communication. Thus the relationship between resting state or task-independent connectivity in specific task networks and behavior is not straightforward and deserves more study before a strong conclusion can be made here. Our pattern of results may also suggest that the DMN is relatively less sensitive to these issues and perhaps represents a network fundamental to the capacity for multiple networks to interact during cognition. For example, one pattern in cognitive aging is the inability for older adults to disengage or deactivate the DMN when task-evoked networks must be recruited (Persson et al., 2007; Miller et al., 2008). This deficit may be related to deficits in flexibly switching recruitment needs among cognitive networks, which reflects what the brain must do to perform well in tasks involving online monitoring and updating, flexibly shifting attention, and task-switching, all components of executive function (Miyake et al., 2000). Our results may therefore help explain why the effects of aerobic exercise training are broadly transferable to executive function relative to other types of aging interventions.

What might be the mechanism for observed connectivity changes resulting from a walking program? A vast animal literature has shown the importance of changes in extracellular neurotrophin levels such as BDNF and NGF (Neeper et al., 1995, 1996) for exercise-induced effects on synaptic plasticity and cognition. In humans, circulating BDNF levels are related to greater hippocampal volume (Erickson et al., 2010), which is in turn related to higher aerobic fitness levels (Erickson et al., 2009). Some studies have also shown an association between neurogenesis and exercise-induced improvement in cognitive performance (Pereira et al., 2007; Creer et al., 2010), yet data also suggest the extent that new neurons in the hippocampus become integrated into distributed systems depends on the level of environmental enrichment one experiences following exercise training (Kempermann, 2008; Fabel et al., 2009). This is consistent with the idea that aerobic exercise promotes the integration of new neurons into existing brain networks, facilitated by the upregulation of trophic factors in the hippocampus and the cerebral cortex, resulting in enhanced cortico-hippocampal connectivity, and that experience modulates the extent to whether and what brain 
regions show increased cortico-hippocampal connectivity. For example, when specific cognitive training is coupled with aerobic training, this may result in a bias toward training-specific changes in connectivity. However, in the absence of a specific form of cognitive training, aerobic training-enhanced connections may occur preferentially in cortico-hippocampal connections implemented often in daily life and shift the pattern of changes to strong anatomical pathways. Our results are consistent with this hypothesis, given the hippocampus has widespread cortical connections with association cortices, including strong connections with the frontal and temporal cortex, and projections to the parietal and lateral occipital cortex through cingulate pathways, and relatively few projections to primary sensory cortices (Miller, 1991). This serves as one potential explanation for the effects observed here, and while speculative, offers a testable prediction for future study.

Regarding differences at the six-month session for the FTB group, it is possible that these effects reflect the specific novel activities that the FTB group experienced (Lewis et al., 2009). For instance, the DMN has been implicated in task states including mind wandering, thought focused inward to the self, and taking the perspective of others into one's own view of the world (Buckner et al., 2008; Schilbach et al., 2008). The FTB intervention was composed of stretching activities to improve flexibility, yoga poses, light strengthening activities, and exercises to improve balance. Cognitively, these activities required participants to imitate an instructor's movements while learning exercises and to maintain focus while holding exercise poses and balance. The activities became sequentially more difficult over the first 6 months of the program and thereafter the more demanding levels were maintained. This gradual progression in difficulty and complexity may explain the two connectivity effects in the DMN in favor of the FTB group at 6 months (see Figure 2).

The other effect in favor of the FTB group, which occurred at 12 months, was increased connectivity in the FP network between the right lateral occipital cortex and the right opercular/insular cortex. Previous characterizations of the cognitive role of the interaction between the FP system and the insular cortex have implicated functions such as reconfiguration of an attentional set to be maintained over a prolonged task state (Dosenbach et al., 2006). Another study showed that active learning was involved in enhancement of connectivity in resting state networks reflective of task-relevant regions in the learned task, while connectivity was not affected by mere rote performance of the same learned task (Albert et al., 2009). Thus we may speculate that the effects in favor of the FTB group at 6 months reflect experience-dependent changes related to the skill of inward mental focus. On the other hand, changes specific to 12 months might reflect a learned capacity to switch among various exercises and redistributing attention to different aspects of the body and environment for different exercises. This suggests the potential of an FTB intervention, combined with other aspects of cognitive training, as a method of combined cognitive and physical training. Along a similar vein, to find the optimal strategy for combined cognitive and physical training, future research should explore the possibility of order effects for treatments (Kempermann, 2008). Any cognitive training addition should also include aspects of training programs that have shown promise for enabling transfer of learned skills to tasks beyond those specifically trained, such as strategic videogames (Basak et al., 2008), social engagement (Carlson et al., 2009), or process specific cognitive training programs for working memory and multi-tasking (Erickson et al., 2007; Dahlin et al., 2008; Karbach and Kray, 2009).

Our results and interpretations must be considered in the context of several limitations. First, our analysis centered around a ROI-based examination of exercise effects on age-sensitive network disruptions. Although this provided a focused examination of the effects of aerobic training on functional connectivity in the context of aging, an important area for future research will be characterizing the multidimensional nature of age-related changes in patterns of brain activation (Cole et al., 2010; Hayasaka and Laurienti, 2010). This line of work could be seen as a complementary, exploratory examination of the effects of exercise on the brain. Another limitation that could be investigated with future research is the inter-play between changes in functional connectivity under no or minimal cognitive demand, connectivity during cognitive demands that stress the FE and the FP systems, and changes in cognitive performance. It will also be important for future research to integrate results from fMRI with other measures of brain structure and function such as structural connectivity, blood flow, electrophysiology, and neurobiological markers of brain function and disease. Finally, for broadest applicability of results, future studies should try to include more diverse participant populations in terms of years of education, gender, and socioeconomic status. These limitations provide a starting point for future research.

Overall this study represents an important extension of previous knowledge of the effects of exercise on the aging brain. We have shown the aging brain is plastic and responsive to changes in exercise behavior, and that this effect is modulated by length of training. This has important implications for bringing scientific findings into practice. These results are by no means definitive, but rather should serve as a starting point for future research on the relationship between exercise training and changes in brain connectivity and cognition. We hope these findings also encourage future use of these methods for examining the efficacy of exercise interventions to improve or maintain brain and cognitive function in cognitively impaired older adults. Perhaps most exciting is the possibility for our results to inform further integration of the animal and human literatures on the effects of exercise on the brain. To date the animal literature has provided a rich characterization of exercise-induced changes in the medial temporal cortex, while the human literature has focused functionally on cortical regions that implement executive functions. The current study suggests that in humans enhanced hippocampal function may facilitate regulation of long-range cortical networks that implement executive control. Specifically how this relates to training-related changes in the magnitude of task-evoked activity is a topic that deserves future research. Further, we provide preliminary evidence for how differential exercise modalities influence communication in brain systems critical to healthy brain aging, and in doing so, provide a new perspective for predicting how exercise affects the aging brain during high-level cognition.

\section{ACKNOWLEDGMENTS}

This work was supported by the National Institute on Aging at the National Institutes of Health (grant numbers R37 AG025667, RO1 AG25032). We would also like to thank Nancy Dodge, Holly Tracy, and the Exercise Psychology Laboratory for their help in data collection. 


\section{REFERENCES}

Adkins, D. L., Boychuk, J., Remple, M. S., and Kleim, J. A. (2006). Motor training induces experience-specific patterns of plasticity across motor cortex and spinal cord. J. Appl. Physiol. 101, $1776-1782$.

Adlard, P.A., Perreau, V. M., and Cotman, C. W. (2005). The exercise-induced expression of BDNF within the hippocampus varies across life-span. Neurobiol. Aging 26, 511-520.

Albert, N. B., Robertson, E. M., and Miall, R. C. (2009). The resting human brain and motor learning. Curr. Biol. 19, 1023-1027.

Andrews-Hanna, J. R., Snyder, A. Z., Vincent, J. L., Lustig, C., Head, D. Raichle, M. E., and Buckner, R.L. (2007). Disruption of large-scale brain systems in advanced aging. Neuron 56 , 924-935.

Barnes, D. E., Yaffe, K., Satariano, W. A., and Tager, I. B. (2003). A longitudinal study of cardiorespiratory fitness and cognitive function in healthy older adults. J. Am. Geriatr. Soc. 51, 459-465.

Basak, C., Boot, W. R., Voss, M. W., and Kramer, A. F. (2008). Can training in a real-time strategy video game attenuate cognitive decline in older adults? Psychol. Aging 23, 765-777.

Beckmann, C. F., Jerome, G. J., and Smith, S. M. (2003). General multilevel linear modeling for group analysis in fMRI. NeuroImage 20, 1052-1063.

Birn, R. M., Diamond, J. B., Smith, M. A., and Bandettini,P.A. (2006). Separating respiratory-variation-related fluctuations from neuronal-activity-related fluctuations in fMRI. NeuroImage 31, 1536-1548.

Black, J. E., Isaacs, K. R., Anderson, B. J., Alcantara, A. A., and Greenough, W. T. (1990). Learning causes synaptogenesis, whereas motor activity causes angiogenesis, in cerebellar cortex of adult rats. Proc. Natl. Acad. Sci. U.S.A. 87, 5568-5572.

Bopp, K. L., and Verhaeghen, P. (2005). Aging and verbal memory span: a meta-analysis. J. Gerontol. B Psychol. Sci. Soc. Sci. 60, P223-P233.

Borg, G. (1985). An Introduction to Borg's RPE-Scale. Ithaca, NY: Mouvement.

Buckner, R. L., Andrews-Hanna, J. R., and Schacter, D. L. (2008). The brain's default network: anatomy, function, and relevance to disease. Ann. N. Y. Acad. Sci. 1124, 1-38.

Carlson, M. C., Erickson, K. I., Kramer, A. F., Voss, M. W., Bolea, N., Mielke, M., McGill, S., Rebok, G. W., Seeman, T., and Fried, L. P. (2009). Evidence for neurocognitive plasticity in at-risk older adults: the experience corps program. J. Gerontol. A Biol. Sci. Med. Sci. 64, 1275-1282.
CDC. (2007). The State of Aging and Health in America 2007. Washington, D.C.: U.S. Department of Health and Human Services, Centers for Disease Control and Prevention.

Chang, C., Cunningham, J. P., and Glover, G.H. (2009). Influence of heart rate on the BOLD signal: the cardiac response function. NeuroImage 44, 857-869.

Chen, K. S., Masliah, E., Mallory, M. and Gage, F. H. (1995). Synaptic loss in cognitively impaired aged rats is ameliorated by chronic human nerve growth factor infusion. Neuroscience $68,19-27$.

Colcombe, S., Erickson, K., Scalf, P., Kim, J., Prakash, R., Mcauley, E., Elavsky, S., Marquez, D., Hu, L., and Kramer, A. (2006). Aerobic exercise training increases brain volume in aging humans. J. Gerontol. A Biol. Sci. Med. Sci. 61, 1166.

Colcombe, S. J., Erickson, K. I., Raz, N., Webb, A. G., Cohen, N. J., McAuley, E., and Kramer, A. F. (2003). Aerobic fitness reduces brain tissue loss in aging humans. J. Gerontol. A Biol. Sci. Med. Sci. 58, 176-180.

Colcombe, S. J., and Kramer, A. F. (2003) Fitness effects on the cognitive function of older adults: a meta-analytic study. Psychol. Sci. 14, 125-130.

Colcombe, S. J., Kramer, A. F., Erickson, K. I., and Scalf, P. (2005). The implications of cortical recruitment and brain morphology for individual differences in inhibitory function in aging humans. Psychol. Aging 20, 363-375.

Colcombe, S. J., Kramer, A. F., Erickson, K I., Scalf, P., McAuley, E., Cohen, N. J., Webb, A., Jerome, G. J., Marquez, D. X. and Elavsky, S. (2004). Cardiovascular fitness, cortical plasticity, and aging. Proc. Natl. Acad. Sci. U.S.A. 101, 3316-3321.

Cole, M. W., Pathak, S., and Schneider, W. (2010). Identifying the brain's most globally connected regions. NeuroImage 49, 3132-3148.

Corbetta, M., and Shulman, G. L. (2002) Control of goal-directed and stimulusdriven attention in the brain. Nat. Rev Neurosci. 3, 215-229.

Cotman, C. W., and Berchtold, N. C. (2002). Exercise: a behavioral intervention to enhance brain health and plasticity. Trends Neurosci. 25 295-301.

Creer, D. J., Romberg, C., Saksida, L. M. van Praag, H., and Bussey, T. J. (2010). Running enhances spatial pattern separation in mice. Proc. Natl. Acad. Sci. U.S.A. 107, 2367-2372.

Dahlin, E., Neely, A. S., Larsson, A. Bäckman, L., and Nyberg, L. (2008). Transfer of learning after updating training mediated by the striatum. Science 320, 1510-1512.
Damoiseaux, J.S., Beckmann, C. F., Arigita E. J. S., Barkhof, F., Scheltens, P., Stam, C. J., Smith, S. M., and Rombouts, S A. R. B. (2008). Reduced resting-state brain activity in the "default network" in normal aging. Cereb. Cortex 18, 1856-1864.

Davis, S. W., Dennis, N. A., Buchler, N. G., White, L. E., Madden, D. J., and Cabeza, R. (2009). Assessing the effects of age on long white matter tracts using diffusion tensor tractography. NeuroImage 46, 530-541.

de Munck, J., Goncalves, S., and Faes, T. (2008). A study of the brain's resting state based on alpha band power, heart rate and fMRI. NeuroImage 42 112-121.

Dennis, N. A., Hayes, S. M., Prince, S. E., Madden, D. J., Huettel, S. A., and Cabeza, R. (2008). Effects of aging on neural correlates of successful item and source memory encoding. J. Exp. Psychol. Learn. Mem. Cogn. 34, 791-808.

Dosenbach, N. U. F., Visscher, K. M. Palmer, E. D., Miezin, F. M., Wenger, K. K., Kang, H. C., Burgund, E. D. Grimes, A. L., Schlaggar, B. L., and Petersen, S. E. (2006). A core system for the implementation of task sets. Neuron 50, 799-812.

Erickson, K. I., Colcombe, S. J., Wadhwa, R., Bherer, L., Peterson, M. S., Scalf, P. E., Kim, J. S., Alvarado, M., and Kramer,A.F. (2007). Training-induced plasticity in older adults: effects of training on hemispheric asymmetry. Neurobiol. Aging 28, 272-283.

Erickson, K., Prakash, R., Voss, M. Chaddock, L., Hu, L., Morris, K., White, S., Wójcicki, T., McAuley, E. and Kramer, A. (2009). Aerobic fitness is associated with hippocampal volume in elderly humans. Hippocampus 19, 1030-1039.

Erickson, K. I., Prakash, R. S., Voss, M. W Chaddock, L. C., Heo, S., McLaren, M., Pence, B. D., Martin, S. A., Vieira, V. J., Woods, J. A., McAuley, E., and Kramer, A. F. (2010). Brain-derived neurotrophic factor is associated with age-related decline in hippocampa volume, J. Neurosci. 30, 5368-5375.

Fabel, K., Wolf, S. A., Ehninger, D., Babu, H., Leal-Galicia, P., and Kempermann, G. (2009). Additive effects of physical exercise and environmental enrichment on adult hippocampal neurogenesis in mice. Front. Neurogenesis 1 , 1-7. doi: 10.3389/neuro.22.002.2009.

Fair, D. A., Schlaggar, B. L., Cohen, A. L. Miezin, F. M., Dosenbach, N. U. F. Wenger, K. K., Fox, M. D., Snyder, A Z., Raichle, M. E., and Petersen, S. E. (2007). A method for using blocked and event-related fMRI data to study "resting state" functional connectivity. NeuroImage 35, 396-405.
Farmer, J., Zhao, X., Van Praag, H., Wodtke, K., Gage, F. H., and Christie, B. R. (2004). Effects of voluntary exercise on synaptic plasticity and gene expression in the dentate gyrus of adult male Sprague-Dawley rats in vivo. Neuroscience 124, 71-79.

Fjell, A., Westlye, L., Amlien, I., Espeseth, T., Reinvang, I., Raz, N., Agartz, I., Salat, D., Greve, D., Fischl, B., Dale, A., and Walhovd, K. (2009). High consistency of regional cortical thinning in aging across multiple samples. Cereb. Cortex 49, 1820-1830.

Fox, M. D., Snyder, A. Z., Vincent, J. L. Corbetta, M., Van Essen, D. C., and Raichle, M. E. (2005). The human brain is intrinsically organized into dynamic, anticorrelated functional networks. Proc. Natl. Acad. Sci. U.S.A. 102, 9673-9678.

Gomez-Pinilla, F., Vaynman, S., and Ying, Z. (2008). Brain-derived neurotrophic factor functions as a metabotrophin to mediate the effects of exercise on cognition. Eur. J. Neurosci. 28, 2278-2287.

Grady, C. L., McIntosh, A. R., and Craik, F. I. M. (2003). Age-related differences in the functional connectivity of the hippocampus during memory encoding. Hippocampus 13, 572-586.

Greicius, M., Srivastava, G., Reiss, A., and Menon, V. (2004). Default-mode network activity distinguishes Alzheimer's disease from healthy aging: evidence from functional MRI. Proc. Natl. Acad. Sci. U.S.A. 101, 4637-4642.

Hampson, M., Driesen, N. R., Skudlarski, P., Gore, J. C., and Constable, R. T. (2006). Brain connectivity related to working memory performance. $J$. Neurosci. 26, 13338-13343.

Hayasaka, S., and Laurienti, P. J. (2010). Comparison of characteristics between region-and voxel-based network analyses in resting-state fMRI data. NeuroImage 50, 499-508.

He, W., Sengupta, M., Velkoff, V. A., and DeBarros, K. A. (2005). 65+ In the United States: 2005. Washington, D.C.: U.S. Census Bureau.

Hedden, T., Van Dijk, K. R. A., Becker, J. A., Mehta, A., Sperling, R. A., Johnson, K. A., and Buckner, R. L. (2009). Disruption of functional connectivity in clinically normal older adults harboring amyloid burden. J. Neurosci. 29, 12686-12694.

Isaacs, K. R., Anderson, B. J., Alcantara, A. A., Black, J. E., and Greenough, W. T. (1992). Exercise and the brain: angiogenesis in the adult rat cerebellum after vigorous physical activity and motor skill learning. J. Cereb. Blood Flow Metab. 12, 110-119.

Jenkinson, M., Bannister, P. R., Brady, J. M., and Smith, S. M. (2002). 
Improved optimisation for the robust and accurate linear registration and motion correction of brain images. NeuroImage 17, 825-841.

Karbach, J., and Kray, J. (2009). How useful is executive control training? Age differences in near and far transfer of task-switching training. Dev. Sci. 12, 978-990.

Kelly, C. A. M., Uddin, L. Q., Biswal, B. B., Castellanos, F. X., and Milham, M. P. (2008). Competition between functional brain networks mediates behavioral variability. NeuroImage $39,527-537$.

Kempermann, G. (2008). The neurogenic reserve hypothesis: what is adult hippocampal neurogenesis good for? Trends Neurosci. 31, 163-169.

Kleim,J.A.,Cooper,N.R., andVandenBerg, P. M. (2002). Exercise induces angiogenesis but does not alter movement representations within rat motor cortex. Brain Res. 934, 1-6.

Kramer, A. F., Hahn, S., and Gopher, D. (1999). Task coordination and aging: explorations of executive control processes in the task switching paradigm. Acta Psychol. 101, 339-378.

Larsen, J. O., Skalicky, M., and Viidik, A. (2000). Does long-term physical exercise counteract age-related Purkinje cell loss? A stereological study of rat cerebellum. J. Comp. Neurol. 428, 213-222.

Larson, E. B., Wang, L., Bowen, J. D., McCormick, W. C., Teri, L., Crane, P., and Kukull, W. (2006). Exercise is associated with reduced risk for incident dementia among persons 65 years of age and older. Ann. Intern. Med. 144, 73-81.

Lewis, C. M., Baldassarre, A., Committeri, G., Romani, G. L., and Corbetta, M. (2009). Learning sculpts the spontaneous activity of the resting human brain. Proc. Natl. Acad. Sci. U.S.A. 106, 17558-17563.

Lindsay, J., Laurin, D., Verreault, R., Hébert, R., Helliwell, B., Hill, G.B., and McDowell, I. (2002). Risk factors for Alzheimer's disease: a prospective analysis from the Canadian Study of Health and Aging. Am. J. Epidemiol. 156, 445-453.

Lustig, C., Snyder, A. Z., Bhakta, M., O'Brien, K. C., McAvoy, M., Raichle, M. E., Morris, J. C., and Buckner, R. L. (2003). Functional deactivations: change with age and dementia of the Alzheimer type. Proc. Natl. Acad. Sci. U.S.A. 100, 14504-14509.

MacRae, P. G., Spirduso, W. W., Cartee, G. D., Farrar, R. P., and Wilcox, R. E. (1987). Endurance training effects on striatal D2 dopamine receptor binding and striatal dopamine metabolite levels. Neurosci. Lett. 79, 138-144.
Madden, D., Spaniol, J., Whiting, W., Bucur, B., Provenzale, J., Cabeza, R., White, L., and Huettel, S. (2007). Adult age differences in the functional neuroanatomy of visual attention: a combined fMRI and DTI study. Neurobiol. Aging 28, 459-476.

McCloskey, D. P., Adamo, D. S., and Anderson, B. J. (2001). Exercise increases metabolic capacity in the motor cortex and striatum, but not in the hippocampus. Brain Res. 891, 168-175.

Miller, R. (1991). Cortico-Hippocampal Interplay, Vol 17. Heidelberg: SpringerVerlag.

Miller, S. L., Celone, K., DePeau, K., Diamond, E., Dickerson, B. C., Rentz, D., Pihlajamaki, M., and Sperling, R A. (2008). Age-related impairment associated with loss of parietal deactivation but preserved hippocampal activation. Proc. Natl. Acad. Sci.U.S.A. 105, 2181-2186.

Miyake, A., Friedman, N. P., Emerson, M. J., Witzki, A. H., Howerter, A., and Wager, T. D. (2000). The unity and diversity of executive functions and their contributions to complex "Frontal Lobe" tasks: a latent variable analysis. Cogn. Psychol. 41, 49-100.

Neeper, S. A., Gómez-Pinilla, F., Choi, J., and Cotman, C. W. (1996). Physical activity increases mRNA for brainderived neurotrophic factor and nerve growth factor in rat brain. Brain Res. 726, 49-56.

NIH. (2007). 2005-2006 Progress Report on Alzheimer's Disease. Washington, D.C.: U.S. Department of Health and Human Services, National Institute on Aging and National Institutes of Health.

O'Sullivan, M., Jones, D. K., Summers, P. E., Morris, R. G., Williams, S. C., and Markus, H. S. (2001). Evidence for cortical "disconnection" as a mechanism of age-related cognitive decline. Neurology 57, 632-638.

Oldfield, R.C. (1971). The assessment and analysis of handedness: the Edinburgh inventory. Neuropsychologia 9 , 97-113.

Park, D. C., and Reuter-Lorenz, P. (2009). The adaptive brain: aging and neurocognitive scaffolding. Annu. Rev. Psychol. 60, 173-196.

Pashler, H. (2000). “Task switching and multi-task performance," in Attention and Performance XVIII: Control of Cognitive Processes, eds S. Monsell and J. Driver (Cambridge: MIT Press), pp. 277-309.

Pereira, A. C., Huddleston, D. E., Brickman, A. M., Sosunov, A. A., Hen, R., McKhann, G. M., Sloan, R., Gage, F. H., Brown, T. R., and Small, S. A. (2007). An in vivo correlate of exercise-induced neurogenesis in the adult dentate gyrus. Proc. Natl. Acad. Sci. U.S.A. 104, 5638-5643.

Persson, J., Lustig, C., Nelson, J. K., and Reuter-Lorenz, P. A. (2007). Age differences in deactivation: a link to cognitive control? J. Cogn. Neurosci. 19, 1021-1032.

Persson, J., Nyberg, L., Lind, J., Larsson, A., Nilsson, L. -G., Ingvar, M., and Buckner, R. L. (2006). Structurefunction correlates of cognitive decline in aging. Cereb. Cortex 16, 907-915.

Raz, N., Lindenberger, U., Rodrigue, K.M., Kennedy, K. M., Head, D., Williamson, A., Dahle, C., Gerstorf, D., and Acker, J.D. (2005). Regional brain changes in aging healthy adults: general trends, individual differences and modifiers. Cereb. Cortex 15, 1676-1689.

Raz, N., Rodrigue, K. M., and Acker, J. D. (2003). Hypertension and the brain: vulnerability of the prefrontal regions and executive functions. Behav. Neurosci. 117, 1169-1180.

Richards, M., Hardy, R., and Wadsworth, M. E. J. (2003). Does active leisure protect cognition? Evidence from a national birth cohort. Soc. Sci. Med. 56, 785-792.

Rushworth, M. F. S., Walton, M. E., Kennerley, S. W., and Bannerman, D. M. (2004). Action sets and decisions in the medial frontal cortex. Trends $\operatorname{Cog} n$. Sci. 8, 410-417.

Schilbach, L., Eickhoff, S. B., RotarskaJagiela, A., Fink, G. R., and Vogeley, K. (2008). Minds at rest? Social cognition as the default mode of cognizing and its putative relationship to the "default system" of the brain. Conscious Cogn 17, 457-467.

Seeley, W. W., Menon, V., Schatzberg, A. F., Keller, J., Glover, G. H., Kenna, H., Reiss, A. L., and Greicius, M. D. (2007). Dissociable intrinsic connectivity networks for salience processing and executive control. J. Neurosci. 27, 2349-2356.

Shehzad, Z., Kelly, A., Reiss, P., Gee, D., Gotimer, K., Uddin, L., Lee, S., Margulies, D., Roy, A., Biswal, B., Petkova, E., Castellanos, F., and Milham, M. (2009). The resting brain: unconstrained yet reliable. Cereb. Cortex 19, 2209-2229.

Sheikh, J. I., and Yesavage, J. A. (1986). "Geriatric depression scale (GDS): recent evidence and development of a shorter version," in Clinical Gerontology: A Guide to Assessment and Intervention, ed. T. L. Brink (New York: The Haworth Press), 165-173.

Smith, S. M. (2002). Fast robust automated brain extraction. Hum. Brain Mapp. 17, 143-155.

Sonntag, W. E., Ramsey, M., and Carter C. S. (2005). Growth hormone and insulin-like growth factor-1 (IGF-1) and their influence on cognitive aging. Ageing Res. Rev. 4, 195-212.

Stern, Y., Sano, M., Paulsen, J., and Mayeux, R. (1987). Modified mini-mental state examination: validity and reliability. Neurology 37, 179.

Strath, S. J., Swartz, A. M., Bassett, D. R., O'Brien, W. L., King, G. A., and Ainsworth, B. E. (2000). Evaluation of heart rate as a method for assessing moderate intensity physical activity. Med. Sci. Sports Exerc. 32, S465-S470.

Swain, R. A., Harris, A. B., Wiener, E. C. Dutka, M.V., Morris, H.D., Theien, B. E., Konda, S., Engberg, K., Lauterbur, P. C., and Greenough, W. T. (2003). Prolonged exercise induces angiogenesis and increases cerebral blood volume in primary motor cortex of the rat. Neuroscience 117, 1037-1046.

Thomas, C., Moya, L., Avidan, G., Humphreys, K., Jung, K. J., Peterson, M. A., and Behrmann, M. (2008). Reduction in white matter connectivity, revealed by diffusion tensor imaging, may account for age-related changes in face perception. J. Cogn Neurosci. 20, 268-284.

van Buuren, M., Gladwin, T., Zandbelt, B., van den Heuvel, M., Ramsey, N., Kahn, R., and Vink, M. (2009). Cardiorespiratory effects on defaultmode network activity as measured with fMRI. Hum. Brain Mapp. 30, 3031-3042.

van Gelder,B.M., Tijhuis,M.A.R., Kalmijn, S., Giampaoli, S., and Kromhout, D. (2007). Decline in cognitive functioning is associated with a higher mortality risk. Neuroepidemiology 28 , 93-100.

Van Praag, H., Christie, B. R., Sejnowski, T. J., and Gage, F. H. (1999a). Running enhances neurogenesis, learning, and long-term potentiation in mice. Proc. Natl. Acad. Sci. U.S.A. 96, 13427-13431.

Van Praag, H., Kempermann, G., and Gage, F. (1999b). Running increases cell proliferation and neurogenesis in the adult mouse dentate gyrus. Nat Neurosci. 2, 266-270.

Van Praag, H., Shubert, T., Zhao, C., and Gage, F. H. (2005). Exercise enhances learning and hippocampal neurogenesis in aged mice. J. Neurosci. 25 , 8680-8685.

Vaynman, S., Ying, Z., and Gomez-Pinilla, F. (2004). Hippocampal BDNF mediates the efficacy of exercise on synaptic plasticity and cognition. Eur. J. Neurosci. 20, 2580-2590.

Vickers, A. J. (2005). Analysis of variance is easily misapplied in the analysis of randomized trials: a critique and discussion of alternative statistical 
discussion of alternative statistical approaches. Psychosom. Med. 67, 652-655.

Vickers, A. J., and Altman, D. G. (2001). Statistics notes: analysing controlled trials with baseline and follow up measurements. BMJ 323, 1123-1124.

Voss, M. W., Erickson, K. I., Chaddock, L., Prakash, R. S., Colcombe, S. J., Morris, K. S., Doerksen, S., Hu, L., McAuley, E., and Kramer, A. F. (2008). Dedifferentiation in the visual cortex: an fMRI investigation of individual differences in older adults. Brain Res. 1244, 121-131.

Voss, M. W., Erickson, K. I., Prakash, R. S., Chaddock, L., Malkowski, E., Alves, H., Kim, J. S., Morris, K. S., White, S. M., Wójcicki, T. R., Hu, L., Szabo, A., Klamm, E., McAuley, E., and Kramer,
A. F. (2010). Functional connectivity: a source of variance in the association between cardiorespiratory fitness and cognition? Neuropsychologia 48, 1394-1406.

Whaley, M. H., Brubaker, P. H., and Otto, R. M. (2006). ACSM's Guidelines for Exercise Testing and Prescription, 7th Edn. New York, NY, Lippincott Williams \& Wilkins.

Wise, R. G., Ide, K., Poulin, M. J., and Tracey, I. (2004). Resting fluctuations in arterial carbon dioxide induce significant low frequency variations in BOLD signal. NeuroImage 21, 1652-1664.

Worsley, K. J., Evans, A. C., Marrett, S., and Neelen, P. (1992). A three dimensional statistical analysis for cbf activation studies in human brain. J. Cereb. Blood Flow Metab. 12, 900-918.
Yaffe, K., Fiocco, A. J., Lindquist, K., Vittinghoff, E., Simonsick, E. M., Newman, A. B., Satterfield, S., Rosano, C., Rubin, S. M., Ayonayon, H. N., Harris, T. B., and Study, H. A. (2009). Predictors of maintaining cognitive function in older adults: the Health ABC study. Neurology 72, 2029-2035.

Zar, J. H. (1996). Biostatistical Analysis, 3rd Edn. Upper Saddle River, NJ: Prentice-Hall.

Conflict of Interest Statement: The authors declare that the research was conducted in the absence of any commercial or financial relationships that could be construed as a potential conflict of interest.

Received: 21 April 2010; paper pending published: 14 May 2010; accepted: 02
July 2010; published online: 26 August 2010.

Citation: Voss MW, Prakash RS, Erickson KI, Basak C, Chaddock L, Kim JS, Alves H, Heo S, Szabo AN, White SM, Wójcicki TR, Mailey EL, Gothe N, Olson EA, McAuley E and Kramer AF (2010) Plasticity of brain networks in a randomized intervention trial of exercise training in older adults. Front. Ag. Neurosci. 2:32. doi: 10.3389/ fnagi.2010.00032

Copyright (ㄷ) 2010 Voss, Prakash, Erickson, Basak, Chaddock, Kim, Alves, Heo, Szabo, White, Wójcicki, Mailey, Gothe, Olson, McAuley and Kramer. This is an open-access article subject to an exclusive license agreement between the authors and the Frontiers Research Foundation, which permits unrestricted use, distribution, and reproduction in any medium, provided the original authors and source are credited. 